\title{
Synchronization and locking in oscillators with flexible periods
}

\author{
by \\ Mariya Alisa Savinov \\ BS in Mathematics and Physics, University of Pittsburgh, 2020 \\ Submitted to the Graduate Faculty of \\ the University Honors College in partial fulfillment \\ of the requirements for the degree of \\ Bachelor of Philosophy
}

University of Pittsburgh

2020 


\title{
UNIVERSITY OF PITTSBURGH \\ UNIVERSITY HONORS COLLEGE
}

This dissertation was presented

by

\author{
Mariya Alisa Savinov
}

It was defended on

March 23rd 2020

and approved by

G. Bard Ermentrout, Department of Mathematics

David Swigon, Department of Mathematics

Jon Rubin, Department of Mathematics

Rachael Neilan, Duquesne University, Department of Mathematics

Dissertation Director: G. Bard Ermentrout, Department of Mathematics 
Copyright (c) by Mariya Alisa Savinov

2020 


\title{
Abstract \\ Synchronization and locking in oscillators with flexible periods
}

\author{
Mariya Alisa Savinov, BPhil
}

University of Pittsburgh, 2020

Upon interaction with a stimulus sequence, an oscillator may assume the stimulus' period via a process called entrainment. Standard models of entrainment assume that the oscillator has a fixed natural period and, thus, a limited range of periods to which it can entrain. However, experiments have shown that some oscillating systems have flexible periods; that is, the period of the oscillator can be changed due to external stimuli, and this period persists when the stimulus is discontinued. Studying this type of coordination, Loehr et al. (2011) showed that the synchronization of pianists with a metronome can be described by a nonlinear oscillator model that is quantitatively described using a circle map of phase and period with sinusoidal coupling terms. Here we introduce two variants, termed the multiplicative and additive forced oscillator models, so-called based on their period descriptions. Unlike the Loehr et al. model, these models include a preferred period, as most biological oscillating systems will oscillate at a fixed natural period when not experiencing driving or damping forces. This study focuses on the stability of points of $N: M$ locking, a complex type of entrainment in which the phase of a model rotates $N$ times in response to $M$ stimuli. Locking types investigated here are 1:1, 1:2, 2:3, along with their reciprocals. We identify numerous parameter regimes of multi-stability, and how such regions evolve with changes in preferred period elasticity. Such multi-stability is not generally possible without a malleable period. The basins of attraction of the various types of N:M locking are investigated, with observations of fractal behavior and remarks on how the domains of attraction depend on coupling and elasticity parameters. Finally, we compare and contrast the multiplicative and additive models with other models of synchronization and beat-keeping. 


\section{Table of Contents}

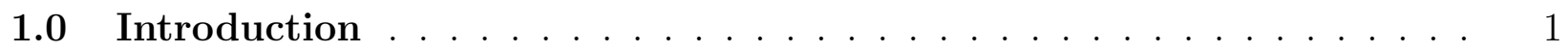

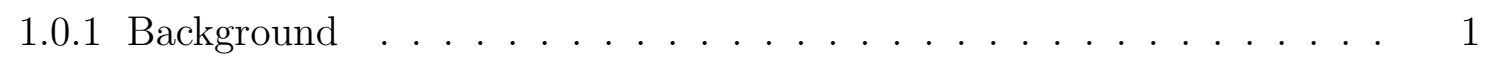

1.0 .2 Standard Oscillator Model . . . . . . . . . . . . . . . . . . 3

1.0 .3 Loehr et al. oscillator model . . . . . . . . . . . . . . . . 8

2.0 Forced Oscillator Models . . . . . . . . . . . . . . . . . . . . 9

$3.0 \quad 1: 1$ Locking - Fixed Points and Stability Conditions . . . . . . . . . . . 11

3.0 .1 1:1 locking - Multiplicative Model . . . . . . . . . . . . . . . . . 11

3.0 .2 1:1 locking - Additive Model . . . . . . . . . . . . . . . . . . . 14

$3.0 .3 \quad 1: 1$ Locking - xppaut AUTO . . . . . . . . . . . . . . . . 17

$\begin{array}{lll}4.0 & 1: 2 \\ 4\end{array}$

$4.0 .1 \quad 1: 2$ locking - Multiplicative Model $\delta=0.0 \ldots \ldots \ldots \ldots$

$4.0 .2 \quad 1: 2$ Locking - Additive Model $\delta=0.0 \ldots \ldots \ldots$

$4.0 .3 \quad 1: 2$ Locking - xppaut AUTO . . . . . . . . . . . . . . 20

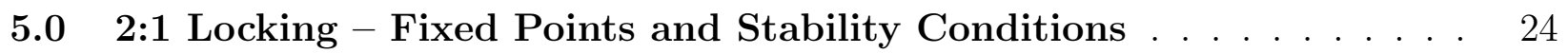

$5.0 .12: 1$ Locking - Multiplicative Model $\delta=0.0 \ldots \ldots \ldots$

$5.0 .2 \quad 2: 1$ Locking - Additive Model $\delta=0.0 \ldots \ldots \ldots$. . . . . . 24

$5.0 .3 \quad 2: 1$ Locking - xppaut AUTO . . . . . . . . . . . . . . . 25

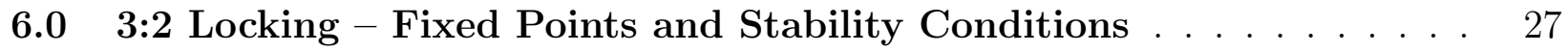

$6.0 .13: 2$ Locking - Multiplicative Model $\delta=0.0 \ldots \ldots \ldots$. . . . 27

$6.0 .2 \quad 3: 2$ Locking - Additive Model $\delta=0.0 \ldots \ldots \ldots \ldots$

$6.0 .3 \quad 3: 2$ Locking -xppaut auto $\ldots \ldots \ldots \ldots \ldots$

7.0 2:3 Locking - xppaut AUTO . . . . . . . . . . . . . . . . . . 32

8.0 Multi-locking parameter regions $\ldots \ldots \ldots \ldots$

9.0 Basins of Attraction $\ldots \ldots \ldots \ldots \ldots$

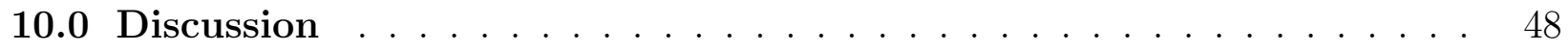

Bibliography . . . . . . . . . . . . . . . . . . . . . . 54 


\subsection{Introduction}

\subsubsection{Background}

Neural oscillations occur when interactions between cells in the central nervous system (neurons) produce repetitive activity. The synchronization of neurons across the brain, in various regions and at various frequencies, is vital to healthy brain behavior and information flow [1]. Neural oscillations are thought to play a key role in processing information and memory [2], and they can even be used as indicators of neurological disorders such as epilepsy or Parkinson's disease, which are characterized by abnormal neural activity [3]. These oscillations are also responsible for many other important global behaviors in organisms. For example, sleep cycles and the body's master-circadian clock are the result of the synchronization of suprachiasmatic nuclei neurons, which are circadian oscillators that operate on a day-night cycle with high activity during the day [1]. In addition, neural oscillations can be indirectly observed in both animals and humans in actions that involve rhythmic motor behavior, such as walking, breathing, or even playing an instrument. Many such actions require coordination with external stimuli (sensorimotor synchronization), particularly those relating to music performance and dance [4]. For example, musicians must synchronize their actions with cues from conductors, metronome beats, or other players. Also, dancers must move in synchrony with musical beats, which requires them to generate temporal expectations [4]. Most studies aiming to understand human beat generation and synchronization have focused on the coordination of simple periodic actions with rhythmic sequences (i.e., tapping to music or discrete sequences). Only recently have studies have been conducted to examine the opposite type of synchronization: the coordination of complex rhythms with simple sequences, i.e. music being played to a metronome [14]. Such studies are relevant to our understanding of musical or rhythmic performance. Another type of coordination that is of interest in such contexts is the coordination of complex rhythms with complex rhythms, such as in duet piano performance and ensemble performance [15, 16, 17, 18]. Such musical

performance requires more complex levels of synchronization and antiphase coordination, so 
it is important to study models which exhibit such complex properties.

Various studies have been conducted to examine and explain the coordination of simple periodic actions with rhythmic sequences. It has been shown that people's intention, attention, and awareness all impact how well they can tap to musical sequences with temporal variability [8, 9]. Also, it has been observed that listeners' coordination of tapping with performed music is impacted by microstructure variations in performance such as timing, intensity, and articulation [5]. The interonset interval (IOI, i.e. stimulus period) range of synchronization for tapping to a metronome has been noted to lie roughly at $100 \mathrm{msec}$ to 1800 msec; the lower limit is reached primarily by musically trained participants, and is a result of both biomechanical limits and the auditory integration window length [4]. Further, it seems that anti-phase tapping has a larger minimum IOI compared to the minimum IOI for in-phase tapping, which makes sense because anti-phase tapping is regarded as more challenging [4].

Two primary types of models have been developed to explain sensorimotor synchronization, some aiming to address properties like those mentioned: error correction models and entrainment (i.e. oscillator) models. Error correction models are based on the idea that the error in the current tap is utilized to adjust the timing of the next tap. A common model utilizes a (phase) correction equation where some $\alpha$ proportion of the expected asynchrony $a_{n}$ is corrected at each step [4, 6].

$$
t_{n+1}=t_{n}-\alpha\left(a_{n}\right)+T_{n}
$$

Here, $t_{n}$ is the expected time of the $n$th tap occurring, $T_{n}$ is the expected timekeeper period just before the $n$th tap, and $a_{n}$ is the expected asynchrony between the $n$th tap and $n$th tone [4, 6]. Though most studies investigating sensorimotor synchronization utilize such error correction models without period correction, there have been studies which utilize a two-way error correction model that includes both phase corrections and period corrections [7]. In the case of Mates (1993), the linear phase correction and linear period correction processes are separate, with phase correction depending on detection of asynchrony and period correction on the difference in periods when comparing to the stimulus [7]. Many 
error correction models use linear terms, but it has been noted that nonlinearity in phase error correction models more accurately reflects phase responses to perturbations [4].

The exclusion of period adaptation terms has been a common theme in entrainment models as well (to be discussed in the following section) [14]. Entrainment models suggest the existence of oscillators, such as those neural oscillations discussed before, to explain coordination and synchronization. Many computational entrainment models, featuring nonlinear terms, have been developed to explain the coordination of periodic actions with rhythmic sequences; these models utilize networks of coupled oscillators, each described at least by their phase [10, 11]. However, entrainment models can also be used to explain the opposite type of synchronization: rhythmic sequences with periodic stimuli.

Aside from error correction and entrainment models, there have been studies which use other formulations to explain beat perception and musical coordination. For example, Bayesian (probabilistic) models have been utilized to analyze the processes behind music analysis and predictive note patterns [12]. Bose et al., on the other hand, utilized a neuromechanistic model to suggest how neuronal networks learn and retain the period and phase of a stimulus [13]. Bose et al.'s neuromechanistic framework operates in the 125 msec to 2000 msec range, which includes the majority of the IOI range which characterizes sensorimotor synchronization [4, 13]. From here, in discussing the synchronization of complex rhythms with simple sequences, we will focus on entrainment models.

\subsubsection{Standard Oscillator Model}

Consider an oscillator with a natural period $T$. The phase of this oscillator $\phi(t)=\frac{t}{T}$ mod 1 gives a measure of the oscillator's progress through its cycle, where $\phi$ is relative to some 0 point (see Fig.1). In the absence of any stimuli, the oscillator will complete a full cycle every $T$ time units without variation. If, however, the oscillator receives a stimulus at time $s \in[0, T]$, the cycle will not be completed at time $T$ but rather at some time $T^{\prime}(s)$. After this point $T^{\prime}(s)$, in the absence of additional stimuli, the oscillator will return to completing cycles in intervals of time length $T$ (see Fig 2). However, if the stimulus is periodic, the oscillator may assume the same period via a process called entrainment. Entrainment is 


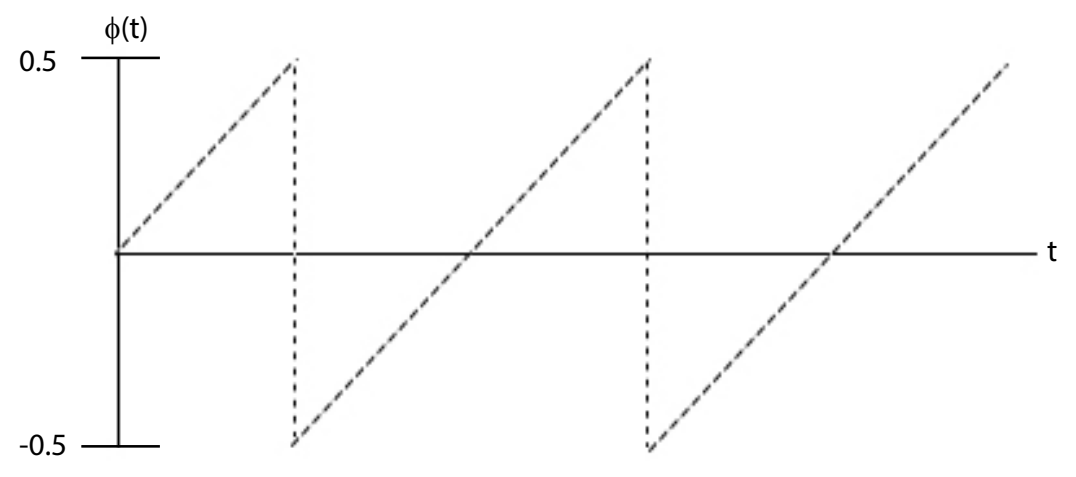

Figure 1: Visual of how an oscillator's phase $\phi(t)$, relative to some 0 , varies with time $t$ : $\phi(t)=\frac{t}{T} \bmod _{[-0.5,0.5]} 1$, where $T$ is the oscillator's natural period.

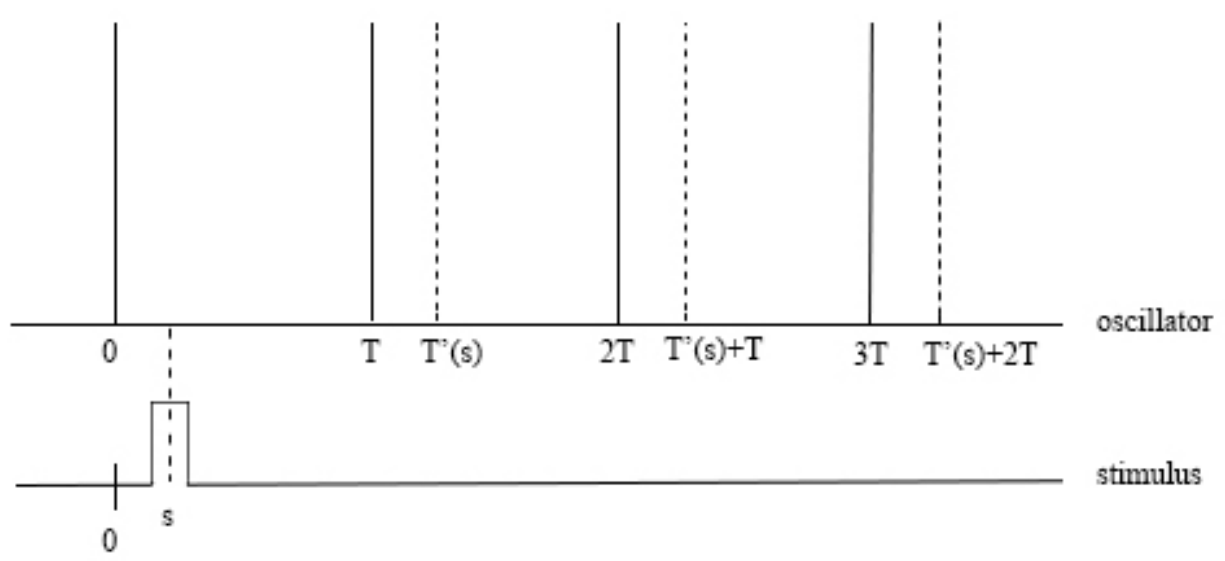

Figure 2: In the absence of stimuli, the oscillator will complete cycles in intervals of $T$ time units, as indicated by the lines at points $0, T, 2 T$, and $3 T$. If, however, the oscillator receives a stimulus at $s \in[0, T]$ time units, as shown by the block on the stimulus line, the oscillator's cycles of offset. The next cycle is completed at time $T^{\prime}(s)$, and then it continues to oscillate at period $T$ in the absence of additional stimuli, as shown by the dotted lines. 
closely related to error correction, discussed above. We can model the oscillator's response to a periodic stimulus of period $P_{n}=t_{n+1}-t_{n}$ using a circle map, where $t_{n}$ is the onset time of stimulus event $n$. The timing difference between the system and stimulus is given by the relative phase $\theta . \theta_{n}$ gives the relative phase representing the phase $\phi$ of the oscillator when an external stimulus event $n$ occurs. From here, we refer to $\theta_{n}$ as simply the phase, though it is important to note that this is relative to the stimulus, not some 0 point.

Here, denoted by " $\theta \bmod { }_{[-0.5,0.5]} 1$ ", we take $\theta \in[-0.5,0.5]$, such that a negative phase $\theta<0$ means the oscillator is late (relative to the stimulus event it was predicting) and a positive phase $\theta>0$ means the model is early (see Fig, 1 for how $\theta$ varies with time $t$ ). Let $\theta_{n}$ and $\hat{\theta}_{n}$ be the phases of the oscillator right before and after the $n$th stimulus. These two phases are related by the following equation set, where the second equation defines the phase response curve:

$$
\left\{\begin{array}{l}
\theta_{n}=\hat{\theta}_{n-1}+\frac{P_{n-1}}{T} \\
\hat{\theta}_{n}=\theta_{n}+\Delta\left(\theta_{n}\right)
\end{array}\right.
$$

Thus we retrieve the standard entrainment model (1.1), where $\Delta\left(\theta_{n}\right)$ defines the coupling between the oscillator and stimulus [19].

$$
\theta_{n+1}=\theta_{n}+\frac{P_{n}}{T}+\Delta\left(\theta_{n}\right)
$$

In terms of frequency, 1.1 can be rewritten as follows, where $\Omega_{n}$ defines the forcing frequency and $\omega$ defines the oscillator frequency:

$$
\theta_{n+1}=\theta_{n}+\frac{\omega}{\Omega_{n}}+\Delta\left(\theta_{n}\right)
$$

This standard model has been utilized to describe various forced oscillator systems. A continuous (in time) version of $(1.2)$, with $\Delta\left(\theta_{n}\right)=\alpha \sin (2 \pi \theta)$, was utilized by Saigusa et al. to describe the periodic entrainment of the plasmodium of Physarum polycephalum (an acellular slime mold) to its environmental conditions [20]. When exposed suddenly to cold and dry environmental conditions, the system, a network of weakly coupled oscillators, exhibits a drastic reduction in locomotion speed. Each oscillator in the network is described by

$$
\frac{\partial \theta}{\partial t}=\omega+\alpha H(t) \sin (2 \pi \theta)+\xi
$$


where $\xi$ is noise and $H(t)=1$ in the presence of a stimulus and $H(t)=0$ under normal conditions. This model exhibits two particular qualities of the P. polycephalum that were experimentally identified: period memorization and recall [20]. Period memorization and recall are valuable qualities of forced oscillators as they are related to learning and memory.

Such standard models of entrainment $(1.1)$ assume that the oscillator has a fixed natural period and, thus, a limited range of periods to which it can entrain [19]. However, experiments have shown that some oscillating systems are able to change their periods [14], and this phenomena has been observed in certain species of fireflies, which exhibit a free-running period [23]. Moreover, some models of coordination have shown that both period and phase adjustment are required to maintain entrainment with temporally variable sequences [10].

A variant of the standard model, which adds period variability, has been used to describe the synchronization of a cell cycle $T_{n}$ to the cellular circadian clock (an example of periodic forcing of a nonlinear oscillator found in biology) [22]. Mosheiff et al. utilized a "kicked cell cycle" model described below, where $t_{n}$ represents the birth time of a cell, $T_{n}$ is the cell cycle duration, $T_{\text {osc }}$ is the forcing period, and $\xi_{n}$ is noise. Unlike the standard model (1.1), an additional dimension is added as a result of the memory term $T_{n-1}$. There is room for the period to vary in each generation, which is unlike both the standard model (1.1) and the Saigusa et al. model 1.3 .

$$
t_{n+1}=t_{n}+\tau_{0}(1-\gamma)+\gamma T_{n-1}+k \sin \left(\frac{2 \pi t_{n}}{T_{o s c}}\right)+\xi_{n}
$$

Here, $\tau_{0}$ is the intrinsic cell-cycle period, $k$ is the strength of cell cycle to circadian oscillator coupling, and $\gamma$ defines mother-daughter coupling. Note that $\xi_{n}$ is noise, so we will ignore it for now $\left(\xi_{n}=0\right)$. Then, this description of the cell cycle can be rewritten in terms of phase $\theta_{n}=\frac{t_{n}}{T_{\text {osc }}}$ to equation 1.4 :

$$
\theta_{n+1}=\theta_{n}+\frac{\tau_{0}}{T_{\text {osc }}}(1-\gamma)+\gamma\left(\theta_{n}-\theta_{n-1}\right)+\tilde{k} \sin \left(2 \pi \theta_{n}\right)
$$

A key additional term, aside from the memory component $\gamma\left(\theta_{n}-\theta_{n-1}\right)$, which separates the Mosheiff et al. model (1.4) from the standard model (1.1) is the term $\frac{\tau_{0}}{T_{\text {osc }}}(1-\gamma)$. This term defines the impact of the natural period of oscillation for the cell, with $(1-\gamma)$ relating to 


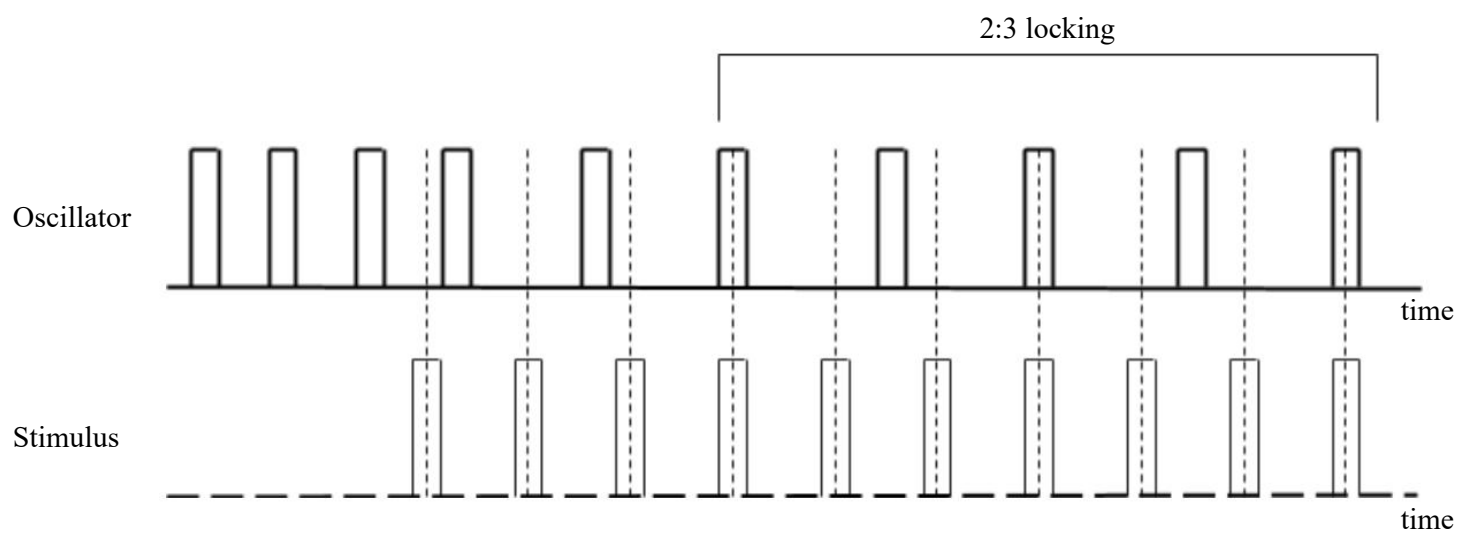

Figure 3: At the end of every rotation, the oscillator "fires", as indicated in the top line by blocks which are initially at some intrinsic period. Once the stimulus is introduced (bottom line), the oscillator synchronizes to a state where it fires twice for three stimuli triggers. This adjusts the oscillator's period and allows it to achieve a state of 2:3 locking.

elasticity. If $\gamma=1$, the model can entrain to any stimulus period $T_{\text {osc }}$; however, a $\gamma \neq 1$ results in a stiffer system about its intrinsic cell-cycle period $\tau_{0}$.

The model suggests that, dependent upon the choices of parameters, coupling to the circadian clock can either increase or decrease variability in the cell-cycle duration of a population of cells [22]. More importantly, for low forcing amplitudes $k$, this model (1.4) exhibits Arnold tongues, which are zones of stable locking, particularly those which are beyond 1:1. The existence and stability of $N: M$ locking, a complex form of entrainment in which the phase $\theta_{n}$ rotates $\mathrm{N}$ times for $\mathrm{M}$ stimuli, is desired for models with a variable period. Fig. 3 shows an example of how an oscillator reaches a state of 2:3 locking, where it "fires" at each completion of a rotation and thus completes two rotations for three stimuli. 


\subsubsection{Loehr et al. oscillator model}

Studying the coordination of complex rhythms with simple sequences, Loehr et al. investigated pianists' responses when playing simple rhythmic pieces to varying metronome sequences [14]. Generally, the sequence would begin at a stable IOI and then, partway through the sequence, the IOI would increase or decrease linearly at a scale of $1 \%$ or $3 \%$ of the initial IOI. Asymmetric asynchrony measurements were observed when comparing the sequences which increased to those which decreased. This asynchrony best described by a nonlinear discrete oscillator model [14]. The Loehr et al. model shares certain properties of neural oscillations, and, unlike standard models (1.1), this oscillator has the ability to change its period of oscillation as well as shift its timing.

The Loehr et al. oscillator (1.5) is quantitatively described using a circle map defined by phase $\theta_{n}$ and period $p_{n}$, where $n$ is a particular event (i.e. a metronome beat). The stimulus period is defined as the interonset interval $I O I_{n}$, equivalent to $P_{n}$ in the standard model (1.1) $\left(P_{n}=t_{n+1}-t_{n}\right.$, where $t_{n}$ is the onset time of an event $\left.n\right)$.

$$
\left\{\begin{array}{l}
\theta_{n+1}=\theta_{n}+\frac{P_{n}}{p_{n}}-\frac{\alpha}{2 \pi} \sin \left(2 \pi \theta_{n}\right) \quad \bmod { }_{[-0.5,0.5]} 1 \\
p_{n+1}=p_{n}\left(1+\frac{\beta^{*}}{2 \pi} \sin \left(2 \pi \theta_{n}\right)\right)
\end{array}\right.
$$

This model utilizes two sinusoidal coupling terms: $\alpha$, the phase coupling strength, and $\beta^{*}$, the period adaptation strength, between the oscillator and stimulus sequence. As previously described, $\theta$ is taken modulus 1 and normalized to be in $[-0.5,0.5]$ so that $\theta_{n}<0$ means the stimulus event $n$ occurred before the model's prediction and $\theta_{n}>0$ means that the stimulus event $n$ occurred after the model's prediction. If $\theta_{n}=0$, then the model is perfectly

synchronized with the stimulus. The intuition behind the period coupling term $\frac{\beta}{2 \pi} \sin 2 \pi \theta_{n}$ is that if the oscillator's prediction is early $\left(\theta_{n}>0\right)$, it lengthens its period (and vice versa, if the oscillator is late, it shortens its period) in order to try to both period and phase lock with the stimulus sequence.

This study focuses on the analysis of two oscillator models which are based on the Loehr et al. model (1.5). Utilizing numerical and analytical techniques, in the next few sections we focus on the stability of fixed points of $N: M$ locking, with particular interest in 1:1, 1:2, $2: 1,2: 3$, and $3: 2$ locking. 


\subsection{Forced Oscillator Models}

The Loehr et al. oscillator model $(1.5)$ is different from standard models of entrainment (1.1) because it has a free-running period. Also, this adaptable period does not feature a preferred period component. Many biological systems have a natural, preferred period at which they will oscillate in the absence of external stimuli. The model (1.5) is also unusual compared to 1.1 because the period coupling term is multiplied by the oscillator's period $p_{n}$, making the coupling strength at each step proportional to $p_{n}$. To address these differences from standard models, we propose two variations of the Loehr et al. oscillator model, with the following period descriptions:

$$
\left\{\begin{array}{l}
A: \quad p_{n+1}=p_{n}\left(1+\frac{\beta^{*}}{2 \pi} \sin \left(2 \pi \theta_{n}\right)\right)+\delta\left(\widetilde{P}-p_{n}\right) \\
B: \quad p_{n+1}=p_{n}+\frac{\beta^{*}}{2 \pi} \sin \left(2 \pi \theta_{n}\right)+\delta\left(\widetilde{P}-p_{n}\right)
\end{array}\right.
$$

$A$ is the multiplicative model, and $B$ is the additive model. Note that when $\delta=0$, the multiplicative model $A$ is defined exactly as the Loehr et al. oscillator model (1.5). In $A$ and $B, \widetilde{P}$ is the natural period of the oscillator. The elasticity $\delta$ determines the effect of $\widetilde{P}$, such that a larger $\delta$ corresponds to a stiffer system. It is important to note that the same $\beta^{*}$ will have vastly different effects on $A$ and $B$, due to the period coupling term in the multiplicative model $\frac{p_{n} \beta^{*}}{2 \pi} \sin \left(2 \pi \theta_{n}\right)$ depending on the magnitude of $p_{n}$. In order to properly compare the variations, it is necessary to scale by $\widetilde{P}$ to a reference frame in which the period coupling terms are of the same magnitude.

Therefore, instead of $p_{n}$, consider a circle map described by a period scaled relative to the preferred period: $q_{n}=\frac{p_{n}}{\widetilde{P}}$. For the additive model $B$, the period coupling term becomes $\beta=\beta^{*} / \widetilde{P}$, while for the multiplicative model $\beta=\beta^{*}$. The interonset interval is also scaled, such that $\widehat{P_{n}}=\frac{P_{n}}{\widetilde{P}}$. Thus, when $\widehat{P_{n}}=1$, the stimulus interonset interval equals the preferred 
period of the oscillator model, $\widetilde{P}$. In full, the multiplicative model is defined as:

$$
\left\{\begin{array}{l}
\theta_{n+1}=\theta_{n}+\frac{\widehat{P_{n}}}{q_{n}}-\frac{\alpha}{2 \pi} \sin \left(2 \pi \theta_{n}\right) \quad \bmod { }_{[-0.5,0.5]} 1 \\
q_{n+1}=q_{n}\left(1+\frac{\beta}{2 \pi} \sin \left(2 \pi \theta_{n}\right)\right)+\delta\left(1-q_{n}\right)
\end{array}\right.
$$

The additive model is defined, then, as:

$$
\left\{\begin{array}{l}
\theta_{n+1}=\theta_{n}+\frac{\widehat{P_{n}}}{q_{n}}-\frac{\alpha}{2 \pi} \sin \left(2 \pi \theta_{n}\right) \quad \bmod _{[-0.5,0.5]} 1 \\
q_{n+1}=q_{n}+\frac{\beta}{2 \pi} \sin \left(2 \pi \theta_{n}\right)+\delta\left(1-q_{n}\right)
\end{array}\right.
$$

For this study, $\alpha$ and $\beta$ are positive parameters within the range $[0,2]$, as otherwise the maps may become non-invertible and large coupling factors are not desirable. $\delta$ is typically kept small (close to zero), as large $\delta$ would not give the systems enough freedom to entrain beyond values close to their preferred period. 


\subsection{1:1 Locking - Fixed Points and Stability Conditions}

Recall that $N: M$ locking is a form of synchronization in which the phase $\theta_{n}$ of an oscillator rotates $N$ times for $M$ stimuli (see Fig. 3 for an example). When in a stable state of N:M locking, an oscillator model will have a period that is roughly a fraction $M / N$ of the stimulus

period $\widehat{P}$, and will exhibit a rotation number of roughly $N / M$. For the multiplicative model (2.1) and the additive model (2.2), we focus on determining the stability of fixed points of 1:1, $1: 2,2: 1,2: 3$, and $3: 2$, with emphasis on how the stability conditions depend on parameters $\alpha, \beta, \delta$, and the stimulus period $\widehat{P}$. Analytical methods are primarily used for the $\delta=0.0$ cases, and numerical software xppaut (AUTO function) 24] is used otherwise.

\subsubsection{1:1 locking - Multiplicative Model}

The multiplicative model 2.1 in the $\delta=0.0$ case is simply a period scaled version of the Loehr et al. model. To determine fixed points of 1:1 locking, we consider the case in which the forcing period is time-independent (i.e., $\widehat{P_{n}}=\widehat{P}$. A fixed point $(\bar{\theta}, \bar{q})$ of 1:1 locking should satisfy the following criteria:

$$
\left\{\begin{array}{l}
\bar{\theta}=\bar{\theta}+\frac{\widehat{P}}{\bar{q}}-\frac{\alpha}{2 \pi} \sin (2 \pi \bar{\theta})-1 \\
\bar{q}=\bar{q}\left(1+\frac{\beta}{2 \pi} \sin (2 \pi \bar{\theta})\right)
\end{array}\right.
$$

There are two solutions to this equation set: $(\bar{\theta}, \bar{q})=(0, \widehat{P})$ and $=(1 / 2, \widehat{P})$. We can determine the stability of these fixed points by linearizing (2.1) about the fixed point $(\bar{\theta}, \bar{q})$, to find the Jacobian:

$$
J_{1, \delta=0}=\left[\begin{array}{cc}
1-\alpha \cos (2 \pi \bar{\theta}) & -\frac{\widehat{P}}{\bar{q}^{2}} \\
\bar{q} \beta \cos (2 \pi \bar{\theta}) & 1+\frac{\beta}{2 \pi} \sin (2 \pi \bar{\theta})
\end{array}\right]
$$


Generally, for such a Jacobian, stability requires that the determinant $\Delta_{1, \delta=0}$ and trace $\tau_{1, \delta=0}$ of $J_{1}$ satisfy the Jury stability criterion $3.2[25]$.

$$
\left\{\begin{array}{l}
|\Delta|<1 \\
|\tau|<\Delta+1
\end{array}\right.
$$

Utilizing these conditions, one can show that, in order for the $1: 1$ fixed point $(0, \widehat{P})$ to be stable, $\alpha$ and $\beta$ must satisfy:

$$
0<\alpha-\beta<2
$$

Note all of the parameters are positive and real, so we omit the additional condition $0<\beta$. The $(1 / 2, \widehat{P})$ fixed point, on the other hand, is always unstable, as stability requires $\beta$ be negative.

The above result (3.3) is a bit peculiar, as intuition would suggest that a larger $\beta$ should 'increase' the stability of 1:1 locking in the oscillator model. However, $\alpha-\beta>0$ indicates that the value of $\alpha$ limits which values $\beta$ can assume. Numerical findings agree with this stability condition found analytically.

Now, let us consider a fixed point of $(\bar{\theta}, \bar{q})$ of the multiplicative model (2.1) when $\delta$ is nonzero. For small $\delta$, we expect that any stability conditions should agree with the $\delta=0$ case (3.3). Assuming again that the forcing period is time-independent, a fixed point of 1:1 locking $(\bar{\theta}, \bar{q})$ must satisfy:

$$
\left\{\begin{array}{l}
\bar{\theta}=\bar{\theta}+\frac{\widehat{P}}{\bar{q}}-\frac{\alpha}{2 \pi} \sin (2 \pi \bar{\theta})-1 \\
\bar{q}=\bar{q}\left(1+\frac{\beta}{2 \pi} \sin (2 \pi \bar{\theta})\right)+\delta(1-\bar{q})
\end{array}\right.
$$

Rearranged, $(\bar{\theta}, \bar{q})$ must satisfy:

$$
\left\{\begin{array}{l}
\frac{1}{2 \pi} \sin (2 \pi \bar{\theta})=\frac{\delta(\widehat{P}-1)}{\beta \widehat{P}+\delta \alpha} \\
\bar{q}=\frac{\beta \widehat{P} / \alpha+\delta}{\beta / \alpha+\delta}
\end{array}\right.
$$

These conditions imply that the existence of a fixed point depends on

$$
\left|\frac{\delta(\widehat{P}-1)}{\beta \widehat{P}+\delta \alpha}\right|<\frac{1}{2 \pi}
$$


As such, in order for 1:1 locking to exist for the case of non-zero $\delta$, the stimulus period $\widehat{P}$ and parameters $\alpha, \beta, \delta$ must satisfy:

$$
\left\{\begin{array}{l}
\widehat{P}>\frac{\delta(2 \pi-\alpha)}{2 \pi \delta+\beta} \\
\widehat{P}<\frac{\delta(2 \pi+\alpha)}{2 \pi \delta-\beta} \text { when } \frac{\beta}{2 \pi \delta}<1 \\
\widehat{P}>\frac{\delta(2 \pi+\alpha)}{2 \pi \delta-\beta} \text { when } \frac{\beta}{2 \pi \delta}>1
\end{array}\right.
$$

Notice if $\frac{\beta}{2 \pi \delta}>1$, there is no upper limit on $\widehat{P}$ for which fixed points of $1: 1$ locking exist. This parameter condition can easily be satisfied when $\delta$ is small as compared to $\beta$. Moreover, when $\delta=0$, these conditions indicate that any parameter set will yield 1:1 locking, as expected for a model with no preferred period $(\delta=0.0)$. Note also that if $\beta=0$, we revert to the conditions for a circle map with an intrinsic period and phase coupling.

We can simplify the conditions of (3.4) in the limit of small $\delta$. Approximating $\delta$ to be close to 0 , a $1: 1$ locking fixed point $(\bar{\theta}, \bar{q})$ should satisfy:

$$
\left\{\begin{array}{l}
\frac{1}{2 \pi} \sin (2 \pi \bar{\theta})=\frac{\delta(\widehat{P}-1)}{\beta \widehat{P}} \\
\bar{q}=\widehat{P}\left(1-\frac{\delta \alpha}{\beta}\right)+\frac{\delta \alpha}{\beta}
\end{array}\right.
$$

Notice that as $\delta \longrightarrow 0, \bar{q} \longrightarrow \widehat{P}$ and $\sin (2 \pi \bar{\theta}) \longrightarrow 0$ or $1 / 2$, which are exactly the fixed points in the $\delta=0$ case discussed previously.

For a fixed point $(\bar{\theta}, \bar{q})$ of $1: 1$ locking (in the nonzero $\delta$ case) of the multiplicative model 2.1 , one can determine stability once again through linearization:

$$
J_{1}=\left[\begin{array}{cc}
1-\alpha \cos (2 \pi \bar{\theta}) & -\frac{\widehat{P}}{\bar{q}^{2}} \\
\beta \bar{q} \cos (2 \pi \bar{\theta}) & 1+\frac{\beta}{2 \pi} \sin (2 \pi \bar{\theta})-\delta
\end{array}\right]
$$

Note that as $\delta \longrightarrow 0, J_{1} \longrightarrow J_{1, \delta=0}\left(3.1\right.$, as expected. Approximating for $\delta$ near zero, $J_{1}$ is approximately:

$$
J_{1} \approx\left[\begin{array}{cc}
1-\alpha & -\frac{1}{\widehat{P}}\left(1-\frac{2 \alpha \delta}{\beta \widehat{P}}(1-\widehat{P})\right) \\
\beta\left(\widehat{P}+\frac{\alpha \delta}{\beta}(1-\widehat{P})\right. & 1-\frac{\delta}{\widehat{P}}
\end{array}\right]
$$


Relating once more back to $J_{1, \delta=0}(0, \widehat{P})$, given by (3.1), $J_{1}$ is simply:

$$
J_{1} \approx J_{1, \delta=0}+\left[\begin{array}{cc}
0 & \frac{2 \alpha \delta}{\beta \widehat{P}}(1-\widehat{P}) \\
\alpha \delta(1-\widehat{P}) & -\frac{\delta}{\widehat{P}}
\end{array}\right]
$$

This reinforces that as $\delta \longrightarrow 0, J_{1} \longrightarrow J_{1, \delta=0}$.

The stability of 1:1 fixed points for the multiplicative model 2.1 in the approximation of small $\delta$ requires that the trace and determinant satisfy 3.2 ). Thus, in order for $(\bar{\theta}, \bar{q}$ ) (for small $\delta$ ) to be stable, the parameters must satisfy the following condition:

$$
0<\alpha-\beta+\delta / \widehat{P}-\alpha \delta<2
$$

As $\delta \longrightarrow 0$, we recover the stability conditions $(3.3)$ of the $(0, \bar{P})$ fixed point for the multiplicative model in the $\delta=0$ case.

\subsubsection{1:1 locking - Additive Model}

Consider the additive model 2.2 in the simplest case: when $\delta=0.0$. Considering the case when the forcing period is time-independent (i.e., $\widehat{P_{n}}=\widehat{P}$ ), a fixed point of 1:1 locking $(\bar{\theta}, \bar{q})$ for 2.2$)$ would satisfy the following criteria:

$$
\left\{\begin{array}{l}
\bar{\theta}=\bar{\theta}+\frac{\widehat{P}}{\bar{q}}-\frac{\alpha}{2 \pi} \sin (2 \pi \bar{\theta})-1 \\
\bar{q}=\bar{q}+\frac{\beta}{2 \pi} \sin (2 \pi \bar{\theta})
\end{array}\right.
$$

Similarly to the multiplicative model, the two solutions to this equation set are $(\bar{\theta}, \bar{q})=(0, \widehat{P})$ and $=(1 / 2, \widehat{P})$. Linearizing about a fixed point, we find that the system Jacobian is:

$$
J_{2, \delta=0}=\left[\begin{array}{cc}
1-\alpha \cos (2 \pi \bar{\theta}) & -\frac{\widehat{P}}{\bar{q}^{2}} \\
\beta \cos (2 \pi \bar{\theta}) & 1
\end{array}\right]
$$

The stability of $(\bar{\theta}, \bar{q})$ is dependent upon the determinant and trace satisfying (3.2). For the $1: 1$ locking fixed point $(0, \widehat{P})$, given that the parameters are positive, we find that the condition for stability is:

$$
0<\alpha-\beta / \widehat{P}<2
$$


The other 1:1 fixed point of the additive model for $\delta=0$ case is $(\bar{\theta}, \bar{q})=(1 / 2, \widehat{P})$. Due to the condition of the parameters being positive, this fixed point is always unstable.

Now, consider the nonzero $\delta$ case of the additive model (2.2). A 1:1 fixed point $(\bar{\theta}, \bar{q})$ must satisfy:

$$
\left\{\begin{array}{l}
\bar{\theta}=\bar{\theta}+\frac{\widehat{P}}{\bar{q}}-\frac{\alpha}{2 \pi} \sin (2 \pi \bar{\theta})-1 \\
\bar{q}=\bar{q}+\frac{\beta}{2 \pi} \sin (2 \pi \bar{\theta})+\delta(1-\bar{q})
\end{array}\right.
$$

Given the above, and that $\sin (2 \pi \bar{\theta}) \in[-1,1]$, the stimulus period $\widehat{P}$ must satisfy the following conditions for fixed points of 1:1 locking to exist for the additive model:

$$
\left(1-\frac{1}{2 \pi} \alpha\right)\left(1-\frac{\beta}{2 \pi \delta}\right)<\widehat{P}<\left(1+\frac{1}{2 \pi} \alpha\right)\left(1+\frac{\beta}{2 \pi \delta}\right)
$$

As $\delta \longrightarrow 0$, the range of stimulus periods for which locking can occur ranges from 0 to $\infty$-this agrees with the $\delta=0.0$ case of the additive model, which has no preferred period. Moreover, this range of locking shows very effectively that $\alpha$ and $\beta$ both increase the range of possible stimuli to which the additive oscillator model can entrain to. This is exactly what we expect to occur as you increase the magnitude of (positive) phase and period coupling terms.

For small $\delta$ (i.e., close to zero), we can approximate the conditions on fixed points $(\bar{\theta}, \bar{q})$ of 1:1 locking to:

$$
\left\{\begin{array}{l}
\bar{q}=\widehat{P}\left(1+\frac{\delta \alpha}{\beta}(1-\widehat{P})\right) \\
\frac{1}{2 \pi} \sin (2 \pi \bar{\theta})=-\frac{\delta}{\beta}(1-\widehat{P})
\end{array}\right.
$$

These conditions imply that the existence of a fixed point depends on

$$
\left|\frac{\delta}{\beta}(1-\widehat{P})\right|<\frac{1}{2 \pi}
$$

This suggests that the range of $\widehat{P}$ for which 1:1 locking can occur (in the case of small $\delta$ ) is:

$$
1-\frac{\beta}{2 \pi \delta}<\widehat{P}<1+\frac{\beta}{2 \pi \delta}
$$

Notice that as $\delta \longrightarrow 0$, the lower and upper limits of locking for $\widehat{P}$ approach $-\infty$ and $+\infty$, respectively, as we would expect for an oscillator with no preferred period. However, contrasting this with the conditions found previously suggests that, when $\delta$ is small, the 
range of 1:1 locking depends almost entirely on the ratio of the period coupling strength $\beta$ to the preferred period elasticity term $\delta$. This is peculiar, but numerical results will agree with the following findings that are based upon the same approximation.

For the additive model, $\delta \neq 0$, the stability of a fixed point $(\bar{\theta}, \bar{q})$ of $1: 1$ locking will depend on qualities of the Jacobian:

$$
J_{2}=\left[\begin{array}{cc}
1-\alpha \cos (2 \pi \bar{\theta}) & -\frac{\widehat{P}}{\bar{q}^{2}} \\
\beta \cos (2 \pi \bar{\theta}) & 1-\delta
\end{array}\right]
$$

As expected, as $\delta \longrightarrow 0, J_{2} \longrightarrow J_{2, \delta=0}$. For small $\delta$, utilizing the conditions on fixed points $(\bar{\theta}, \bar{q})$ given by $(3.9), J_{2}$ is approximately:

$$
J_{2} \approx\left[\begin{array}{cc}
1-\alpha & -\frac{1}{\widehat{P}}\left(1-\frac{2 \alpha \delta}{\beta}(1-\widehat{P})\right) \\
\beta & 1-\delta
\end{array}\right]
$$

which, in terms of $J_{2, \delta=0}$, is

$$
J_{2} \approx J_{2, \delta=0}+\left[\begin{array}{cc}
0 & +\frac{2 \alpha \delta}{\beta \widehat{P}}(1-\widehat{P}) \\
0 & -\delta
\end{array}\right]
$$

This emphasizes again that $J_{2}=J_{2, \delta=0}$ when $\delta=0$. In order for $(\bar{\theta}, \bar{q})$ to be a stable fixed point of 1:1 locking, the trace and determinant must satisfy (3.2). These conditions equivalently become:

$$
\left\{\begin{array}{l}
0<\beta / \widehat{P}+\alpha \delta(3-2 / \widehat{P}) \\
0<\alpha-\beta / \widehat{P}-\alpha \delta(3-2 / \widehat{P})+\delta<2
\end{array}\right.
$$

We see once again that if $\delta \longrightarrow 0,3.11$ approaches the stability conditions (3.8). In addition, if we take $\widehat{P}=1$, we see conditions similar to the multiplicative model stability results 3.6 :

$$
0<\alpha-\beta-\alpha \delta+\delta<2
$$




\subsubsection{1:1 Locking - xppaut AUTO}

The boundaries of fixed point stability can be confirmed numerically through the use of xppaut AUTO. We fix the stimulus period to $\widehat{P}=1$, so that it is exactly the preferred period, and set the preferred period elasticity term $\delta$ to $0.0,0.01,0.1$. We then vary $\alpha$ and $\beta$ to find the Hopf bifurcations which match analytical findings (3.6) and (3.11). The figures of the numerical results are omitted, as there were no unexpected stability regions and all findings agreed with analytic results. 


\subsection{1:2 Locking - Fixed Points and Stability Conditions}

For higher levels of locking, it is challenging to determine stability conditions through analytic means, as the exact values of fixed points become dependent on parameter choices $(\alpha, \beta, \delta)$. For N:M locking, we expect to see $2 \mathrm{M}$ fixed points, with scaled periods of roughly $\bar{q} \approx M / N$. For many choices of N:M locking, if $\delta=0.0$, fixed points exist for any parameter set $(\alpha, \beta)$; however, when $\delta \neq 0$, for each $\alpha$ there is a minimum (and at times maximum) $\beta$ at which fixed points exist. As such, analytical focus will be on the $\delta=0.0$ case of both the multiplicative (2.1) and additive (2.2) models. The boundaries of existence and stability for when $\delta \neq 0$ will be found numerically. We will also turn focus to the special case of $\widehat{P}=1$ for all remaining analytical results.

\subsubsection{1:2 locking - Multiplicative Model $\delta=0.0$}

In the case of $\delta=0.0$ for the multiplicative oscillator model, there are four fixed points of 1:2 locking. $(0,2)$ and $(1 / 2,2)$ are fixed points which exist regardless of parameter choice; the other two fixed points are specific to the choice of coupling parameters $\alpha$ and $\beta$. When iterated at a fixed point, the multiplicative model's phase and scaled period $\left(\theta_{n}, q_{n}\right)$ will oscillate between the two fixed points of a given pair. To analytically determine the stability of the fixed point pair $(0,2)$ and $(1 / 2,2)$, consider the product of the Jacobian $J_{1}$ (3.5) at both points:

$$
J_{1}(0,2) \times J_{1}(1 / 2,2)=\left[\begin{array}{cc}
1-\alpha & -\frac{1}{4} \\
2 \beta & 1
\end{array}\right] \times\left[\begin{array}{cc}
1+\alpha & -\frac{1}{4} \\
-2 \beta & 1
\end{array}\right]
$$

Resulting in the total Jacobian:

$$
\left[\begin{array}{cc}
1-\alpha^{2}+\frac{1}{2} \beta & -\frac{1}{2}+\frac{1}{4} \alpha \\
2 \alpha \beta & 1-\frac{1}{2} \beta
\end{array}\right]
$$


Stability requires that the trace and determinant satisfy 3.2 . As such, the conditions for stability of the fixed point pair $(0,2)$ and $(1 / 2,2)$ are given by:

$$
\left\{\begin{array}{l}
2 \alpha^{2}-4<\alpha \beta-\frac{1}{4} \beta^{2} \\
0<\alpha-\frac{1}{4} \beta \\
0<\alpha^{2}-\alpha \beta+\frac{1}{4} \beta^{2}<2
\end{array}\right.
$$

\subsubsection{1:2 Locking - Additive Model $\delta=0.0$}

In the case of $\delta=0.0$ for the additive oscillator model (2.2), there are four fixed points of 1:2 locking. $(0,2)$ and $(1 / 2,2)$ are fixed points which exist regardless of parameter choice, similar to the multiplicative model above. The other two fixed points are specific to the choice of coupling parameters $\alpha$ and $\beta$. When iterated at a fixed point, the additive model's phase and scaled period $\left(\theta_{n}, q_{n}\right)$ will oscillate between the two fixed points of a given pair. To analytically determine the stability of the fixed point pair $(0,2)$ and $(1 / 2,2)$, we consider the product of the Jacobian $J_{2}(3.10)$ at both points:

$$
J_{2}(0,2) \times J_{2}(1 / 2,2)=\left[\begin{array}{cc}
1-\alpha & -\frac{1}{4} \\
\beta & 1
\end{array}\right] \times\left[\begin{array}{cc}
1+\alpha & -\frac{1}{4} \\
-\beta & 1
\end{array}\right]
$$

Resulting in the total Jacobian:

$$
\left[\begin{array}{cc}
1-\alpha^{2}+\frac{1}{4} \beta & -\frac{1}{2}+\frac{1}{4} \alpha \\
\alpha \beta & 1-\frac{1}{4} \beta
\end{array}\right]
$$

Stability requires that the trace and determinant satisfy 3.2 . As such, the conditions for stability of the fixed point pair $(0,2)$ and $(1 / 2,2)$ are given by:

$$
\left\{\begin{array}{l}
2 \alpha^{2}-4<\frac{1}{2} \alpha \beta-\frac{1}{16} \beta^{2} \\
0<\alpha-\frac{1}{8} \beta \\
0<\alpha^{2}-\frac{1}{2} \alpha \beta+\frac{1}{16} \beta^{2}<2
\end{array}\right.
$$




\subsubsection{1:2 Locking - xppaut AUTO}

Recall that for $\delta=0.0$, there are fixed points of 1:2 locking for all coupling parameter choices $\alpha$, and $\beta$. However, this is not the case for $\delta \neq 0.0$. Moreover, the four fixed points, when they exist, are completely dependent upon the choice of $\alpha$ and $\beta$ (in comparison, when $\delta=0.0$, there is a fixed point pair $(0,2),(1 / 2,2)$ that is independent of parameter choice). For the nonzero $\delta$ case in both the additive and multiplicative models, we rely on numerical software xppaut to find the boundaries of existence and stability. Limit points signify the boundaries of existence, and Hopf bifurcations determine stability boundaries. Fig. 4 shows two specific examples of such points, and their effects, when you vary one parameter ( $\alpha$ or $\beta$ ) and observe the effect on the fixed point (represented by its phase $\theta$ value). Finally, branch points determine when stability switches between the fixed point pairs-this is particularly relevant when we look at cases where we know the stability of one pair analytically, but not the other.

For $\delta=0.0,0.01,0.1$, Fig. 5 shows the numerical results, with shaded regions which represent parameters $(\beta, \alpha)$ for which stable fixed points exist. Note that below the limit point curves, no fixed points exist. In the $\delta=0.0$ case, branch points divide the regions which were predicted analytically from solely numerical results (as we did not analytically predict the stability of the fixed point pair which depends upon the choice of $\alpha$ and $\beta$ ). Thus, we see that numerical methods prove just as necessary for the $\delta=0.0$ case as the non-zero $\delta$ case (see top-left plot in Fig. 5).

To detail exactly which curves were expected analytically, we need simply rewrite the stability conditions 4.1) as:

$$
\left\{\begin{array}{l}
\beta \neq 2 \alpha \\
\beta<4 \alpha \\
-2 \sqrt{4-\alpha^{2}}+2 \alpha<\beta<2 \sqrt{4-\alpha^{2}}+2 \alpha
\end{array}\right.
$$

These three inequalities exactly define the two rightmost Hopf-bifurcations and branch point shown in the top-left subplot of Fig. 5. Similarly, for the $\delta=0.0$ additive model case, 4.2 

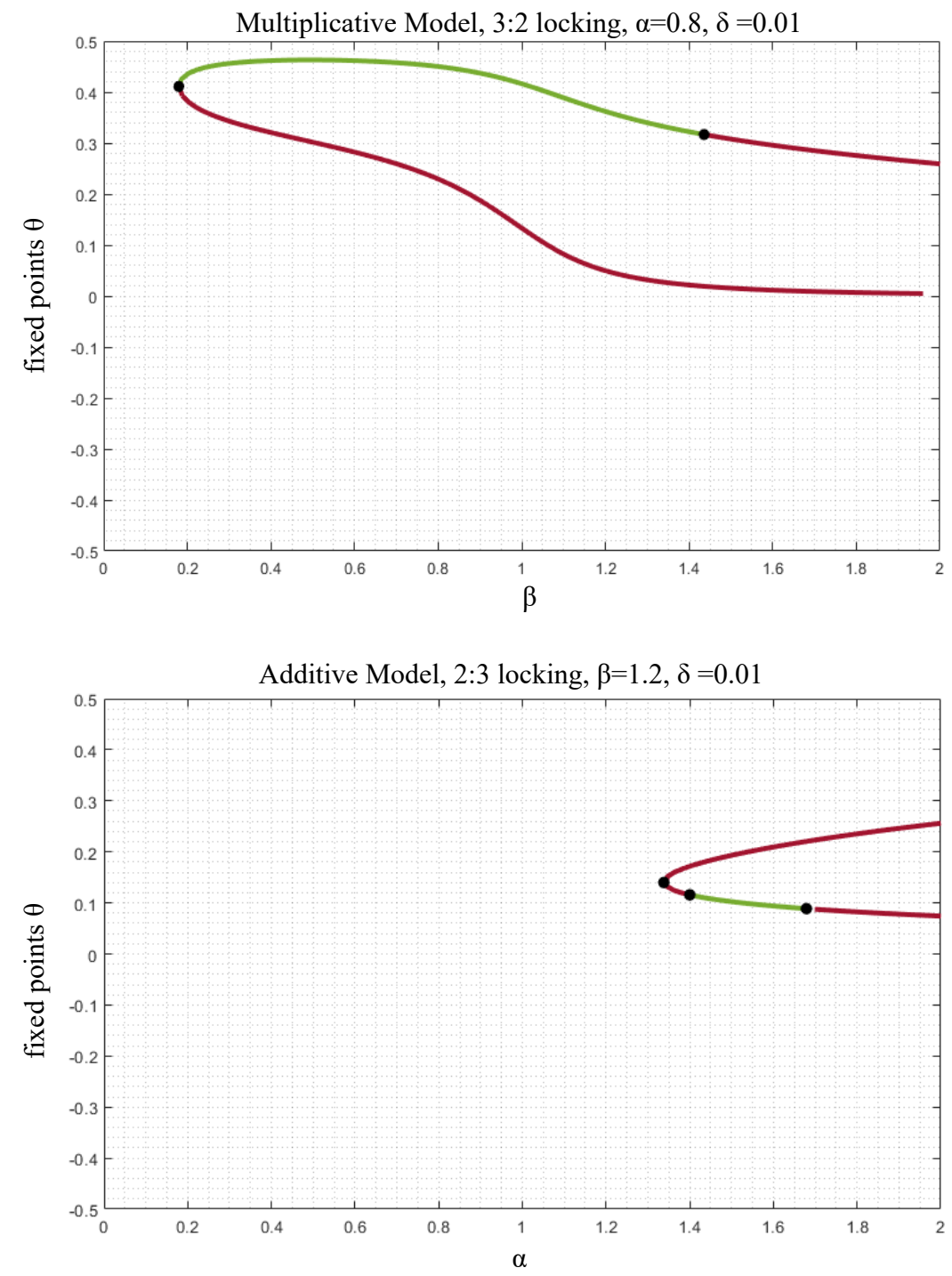

Figure 4: One-parameter plots $(\theta$ v. $\beta$ or $\alpha$ ) showing how the stability and value of fixed points' phase $\theta$ changes when varying a locking parameter. Red lines represent unstable fixed points, while green lines represent stable fixed points. Black points mark either limit points or Hopf bifurcations. In the top plot, the left point is a limit point, and the right point is a Hopf bifurcation. In the bottom plot, the rightmost two points are Hopf bifurcations, and the leftmost point is a limit point. 
can be rewritten in the form:

$$
\left\{\begin{array}{l}
\beta \neq 4 \alpha \\
\beta<8 \alpha \\
-4 \sqrt{4-\alpha^{2}}+4 \alpha<\beta<4 \sqrt{4-\alpha^{2}}+4 \alpha
\end{array}\right.
$$

These curves exactly define the two rightmost Hopf-bifurcations and branch point shown in the top-right subplot of Fig. 5 . 

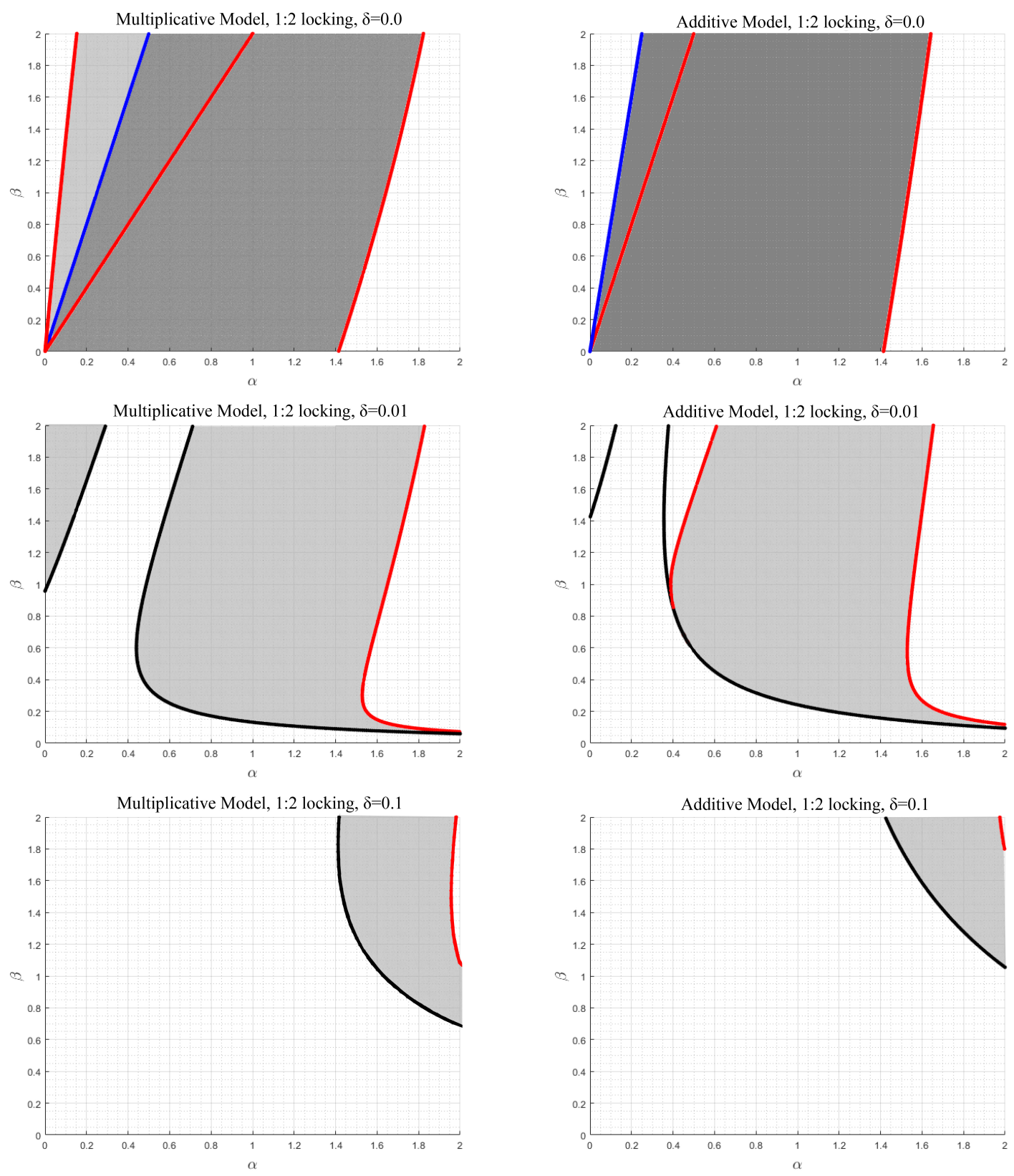

Figure 5: Multi-parameter ( $\beta$ v. $\alpha$ ) plots showing the stability and existence boundaries of 1:2 locking in the multiplicative and additive models as curves in $(\beta, \alpha)$ space. Red curves represent Hopf bifurcations, black curves represent limit points, and blue curves are branch points. Shaded regions represent parameter sets which have stable fixed points. The darker shade of regions in the $\delta=0.0$ case represents that these are regions which were identified analytically as well as numerically. 


\subsection{2:1 Locking - Fixed Points and Stability Conditions}

\subsubsection{2:1 Locking - Multiplicative Model $\delta=0.0$}

For 2:1 locking, the multiplicative oscillator model has two fixed points: $(\bar{\theta}, \bar{q})=(0,1 / 2)$ and $(\bar{\theta}, \bar{q})=(1 / 2,1 / 2)$. Stability of the fixed points can be found by looking at $J_{1}$, given by (3.5), at $(0,1 / 2)$ and $(1 / 2,1 / 2)$ :

$$
J_{1}(0,1 / 2)=\left[\begin{array}{cc}
1-\alpha & -4 \\
\frac{\beta}{2} & 1
\end{array}\right] \quad J_{1}(1 / 2,1 / 2)=\left[\begin{array}{cc}
1+\alpha & -4 \\
-\frac{\beta}{2} & 1
\end{array}\right]
$$

Requiring that the determinant and trace satisfy (3.2), the condition for stability of $(0,1 / 2)$ becomes:

$$
\{0<\alpha-2 \beta<2
$$

Note that here, we exclude conditions that are already satisfied by $\beta>0$ and $\alpha>0$. The fixed point $(1 / 2,1 / 2)$, on the other hand, is always unstable, because it requires a negative $\beta$ for stability.

\subsubsection{2:1 Locking - Additive Model $\delta=0.0$}

For 2:1 locking, the additive oscillator model has two fixed points, similar to the multiplicative model: $(0,1 / 2)$ and $(1 / 2,1 / 2)$. Stability of these fixed points depends upon the trace and determinant of $J_{2}$ (given by $(3.10)$ ) at $(0,1 / 2)$ and $(1 / 2,1 / 2)$ :

$$
J_{2}(0,1 / 2)=\left[\begin{array}{cc}
1-\alpha & -4 \\
\beta & 1
\end{array}\right] \quad J_{2}(1 / 2,1 / 2)=\left[\begin{array}{cc}
1+\alpha & -4 \\
-\beta & 1
\end{array}\right]
$$

Given the constraints that the trace and determinant must satisfy 3.2 in order to have a stable $2: 1$ fixed point, $\alpha$ and $\beta$ must satisfy the following conditions for $(0,1 / 2)$ to be a stable fixed point:

$$
0<\alpha-4 \beta<2
$$


Note again that conditions requiring $\alpha>0$ and $\beta>0$ are omitted, as this is already satisfied by the choice of parameters. The fixed point $(1 / 2,1 / 2)$, on the other hand, is always unstable, because the system requires a negative period coupling term $\beta$ for stability.

\subsubsection{2:1 Locking - xppaut AUTO}

Recall that for $\delta=0.0$, there are two points of 2:1 locking for all coupling parameter choices $\alpha$, and $\beta$. However, this is not the case for $\delta \neq 0.0$. Moreover, the two fixed points, when they exist, are completely dependent upon the choice of $\alpha$ and $\beta$ (in comparison, when $\delta=0.0$, the fixed points' existence is independent of the parameters). For the nonzero $\delta$ case in both the additive and multiplicative models, we rely on numerical software xppaut to find the boundaries of existence and stability. Limit points provide the boundaries of existence, and Hopf bifurcations determine stability boundaries. We expect the shape of the parameter region to be more distorted with larger $\delta$, but to still retain some of the $\delta=0.0$ shape.

For $\delta=0.0,0.01,0.1$, Fig. 5 shows the numerical results, with emphasis on shaded regions which represent parameters $(\beta, \alpha)$ for which stable fixed points exist. Note that below the limit point curves, no fixed points exist. As expected, the larger $\delta$ results in smaller regions of parameter existence and a distortion in the original triangular shape of the $\delta=0.0$ regions given by $(5.1)$ and $(5.2)$. 

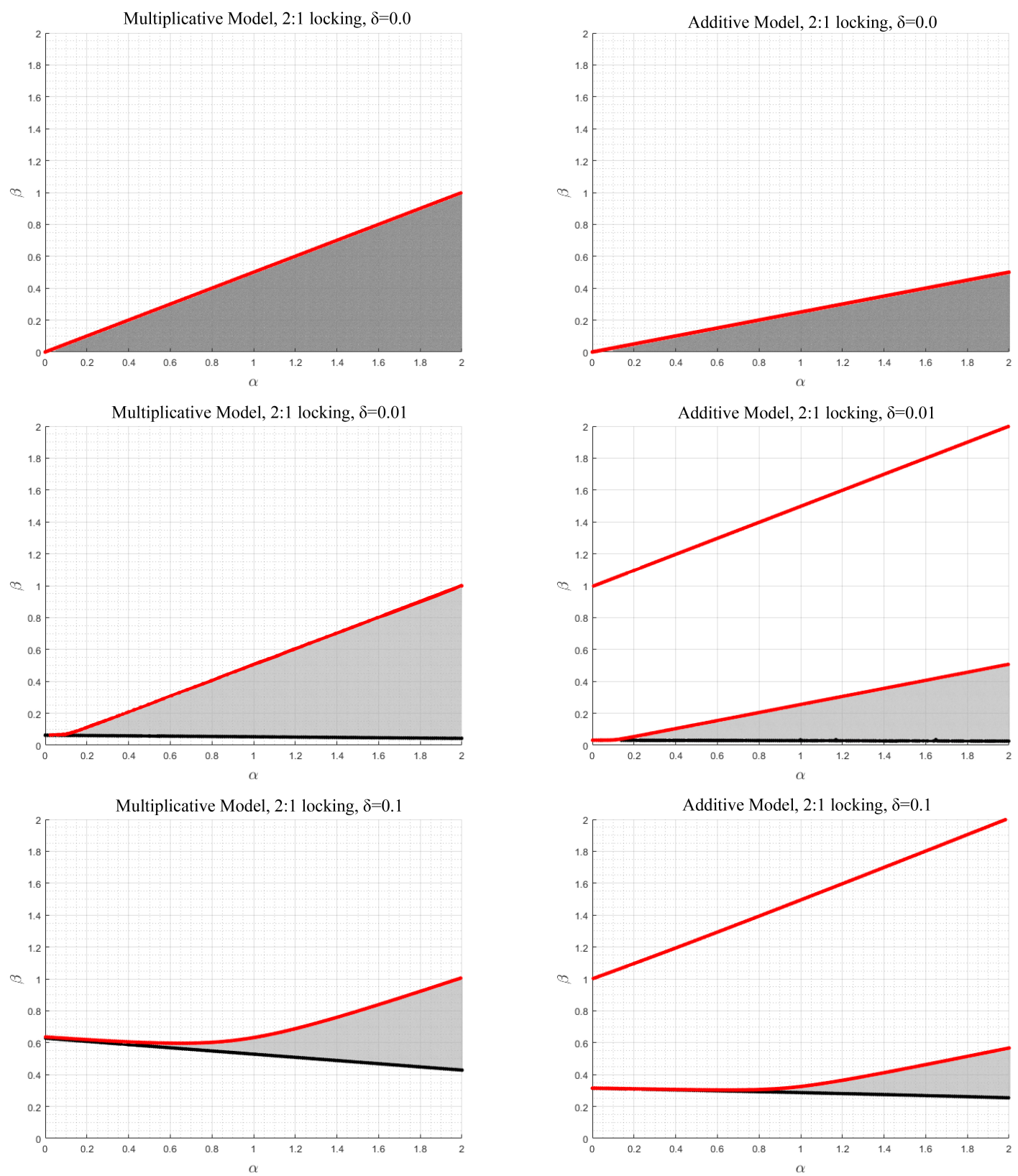

Figure 6: Multi-parameter $(\beta$ v. $\alpha)$ plots showing the stability and existence boundaries of 2:1 locking in the multiplicative and additive models as curves in $(\beta, \alpha)$ space. Red curves represent Hopf bifurcations, and black curves represent limit points. Shaded regions represent parameter sets which have stable fixed points. The darker shade of regions in the $\delta=0.0$ case represents that these are regions which were identified analytically as well as numerically. 


\subsection{3:2 Locking - Fixed Points and Stability Conditions}

The multiplicative (2.1) and additive (2.2) oscillator models have four fixed points of 3:2 locking. Two of these fixed points are independent of parameter choice: $(0,2 / 3)$ and $(1 / 2,2 / 3)$. At a steady state of 3:2 locking, the models' phase and scaled period $\left(\theta_{n}, q_{n}\right)$ will oscillate between the two fixed points of a given pair. However, note that at most one of the pairs of the fixed points, if any, is stable for a given parameter choice.

\subsubsection{3:2 Locking - Multiplicative Model $\delta=0.0$}

For the multiplicative model, determining the stability of the $(0,2 / 3)$ and $(1 / 2,2 / 3)$ fixed point pair requires us to linearize about both points and compute the product of Jacobians, similarly to when determining the stability of pairs of 1:2 locking fixed points. Evaluating $J_{1}$, given by (3.1), at this fixed point pair results in:

$$
J_{1}(0,2 / 3) \times J_{1}(1 / 2,2 / 3)=\left[\begin{array}{cc}
1-\alpha & -\frac{9}{4} \\
\frac{2}{3} \beta & 1
\end{array}\right] \times\left[\begin{array}{cc}
1+\alpha & -\frac{9}{4} \\
-\frac{2}{3} \beta & 1
\end{array}\right]
$$

Thus, the final Jacobian is:

$$
\left[\begin{array}{cc}
1-\alpha^{2}+\frac{3}{2} \beta & -\frac{9}{2}+\frac{9}{4} \alpha \\
\frac{2}{3} \alpha \beta & 1-\frac{3}{2} \beta
\end{array}\right]
$$

In order for the fixed point pair $(0,2 / 3)$ and $(1 / 2,2 / 3)$ to be stable, the above Jacobian's determinant and trace must satisfy 3.2. Rearranging and simplifying these conditions shows us that the $(0,2 / 3),(1 / 2,2 / 3)$ fixed points of $3: 2$ locking are stable if $\alpha$ and $\beta$ satisfy:

$$
\left\{\begin{array}{l}
2 \alpha^{2}-4<3 \alpha \beta-\frac{9}{4} \beta^{2} \\
0<\alpha-\frac{3}{4} \beta \\
0<\alpha^{2}-3 \alpha \beta+\frac{9}{4} \beta^{2}<2
\end{array}\right.
$$




\subsubsection{3:2 Locking - Additive Model $\delta=0.0$}

Recall that the additive model also features four fixed points, including a pair of which is parameter independent for which we will analytically determine stability. The stability of the $(0,2 / 3)$ and $(1 / 2,2 / 3)$ can be found through analysis similar to the multiplicative model case, where we linearize about both points and find the product of the Jacobians. Evaluating $J_{2}$, given by (3.7), at this fixed point pair gives:

$$
J_{2}(0,2 / 3) \times J_{2}(1 / 2,2 / 3)=\left[\begin{array}{cc}
1-\alpha & -\frac{9}{4} \\
\beta & 1
\end{array}\right] \times\left[\begin{array}{cc}
1-\alpha & -\frac{9}{4} \\
\frac{2}{3} \beta & 1
\end{array}\right]
$$

Thus, the final Jacobian is:

$$
\left[\begin{array}{cc}
1-\alpha^{2}-\frac{9}{4} \beta & -\frac{9}{2}-\frac{9}{4} \alpha \\
\alpha \beta & 1+\frac{9}{4} \beta
\end{array}\right]
$$

In order for the fixed point pair $(0,2 / 3)$ and $(1 / 2,2 / 3)$ to be stable, the above Jacobian's determinant and trace must satisfy (3.2). These conditions can be simplified to the following three inequalities:

$$
\left\{\begin{array}{l}
2 \alpha^{2}-4<\frac{9}{2} \alpha \beta-\frac{81}{16} \beta^{2} \\
0<\alpha-\frac{9}{8} \beta \\
0<\alpha^{2}-\frac{9}{2} \alpha \beta+\frac{81}{16} \beta^{2}<2
\end{array}\right.
$$

\subsubsection{3:2 Locking -xppaut auto}

Recall that for $\delta=0.0$, there are fixed points of 3:2 locking for all coupling parameter

choices $\alpha$, and $\beta$. However, this is not the case for $\delta \neq 0.0$. Moreover, the four fixed points, when they exist, are completely dependent upon the choice of $\alpha$ and $\beta$ (in comparison, when $\delta=0.0$, there is a fixed point pair $(0,2 / 3),(1 / 2,2 / 3)$ that is independent of parameter choice). For the nonzero $\delta$ case in both the additive and multiplicative models, we rely on numerical software xppaut to find the boundaries of existence and stability. Limit points provide the boundaries of existence, and Hopf bifurcations determine stability boundaries. 
For $\delta=0.0,0.01,0.1$, Fig. 7 shows the numerical results, with emphasis on shaded regions which represent parameters $(\beta, \alpha)$ for which stable fixed points exist. Note that below and between particular limit point curves, no fixed points exist. In the $\delta=0.0$ case, branch points divide the regions which were predicted analytically from solely numerical results (as we could not predict the stability of the fixed point pair which depended upon the choice of $\alpha$ and $\beta$ ). Thus, we see once again that numerical methods prove just as necessary for the $\delta=0.0$ case as the non-zero $\delta$ case (see top-left subplot in Fig. 7 ).

To detail exactly which curves were expected analytically for the multiplicative model when $\delta=0.0$, we need simply rewrite the stability conditions (6.1) as follows:

$$
\left\{\begin{array}{l}
\beta \neq \frac{2}{3} \alpha \\
\beta<\frac{4}{3} \alpha \\
-\frac{2}{3} \sqrt{4-\alpha^{2}}+\frac{2}{3} \alpha<\beta<\frac{2}{3} \sqrt{4-\alpha^{2}}+\frac{2}{3} \alpha
\end{array}\right.
$$

These curves, put together, describe the darker shaded region shown in Fig. 7, top-left corner. Similarly, for the additive model $\delta=0.0,6.2$ is equivalent to:

$$
\left\{\begin{array}{l}
\beta \neq \frac{4}{9} \alpha \\
\beta<\frac{8}{9} \alpha \\
-\frac{4}{9} \sqrt{4-\alpha^{2}}+\frac{4}{9} \alpha<\beta<\frac{4}{9} \sqrt{4-\alpha^{2}}+\frac{4}{9} \alpha
\end{array}\right.
$$

These curves outline the region shown in Fig. 7 , top-right corner.

For 3:2 locking, we see in Fig. 7 exactly how increasing $\delta$ leads to changes in parameter regions of stable fixed points. As $\delta$ increases, the regions where fixed points exist get smaller, requiring larger $\beta$ than in smaller $\delta$ cases for the same $\alpha$. Further, there seems to be a general shift away from $\alpha=0, \beta=0$, such that the regions of stable 3:2 fixed points require both a large $\beta$ and large $\alpha$ for larger $\delta$. For example, when $\delta=0.1$ for the additive model (bottom left of Fig. 7), $\alpha$ must be larger than 1.8 and $\beta$ must be larger than 0.5. Such large values for the phase coupling strength are not desirable, but are clearly required to achieve 3:2 locking for a model with a 'strong' preferred period. 

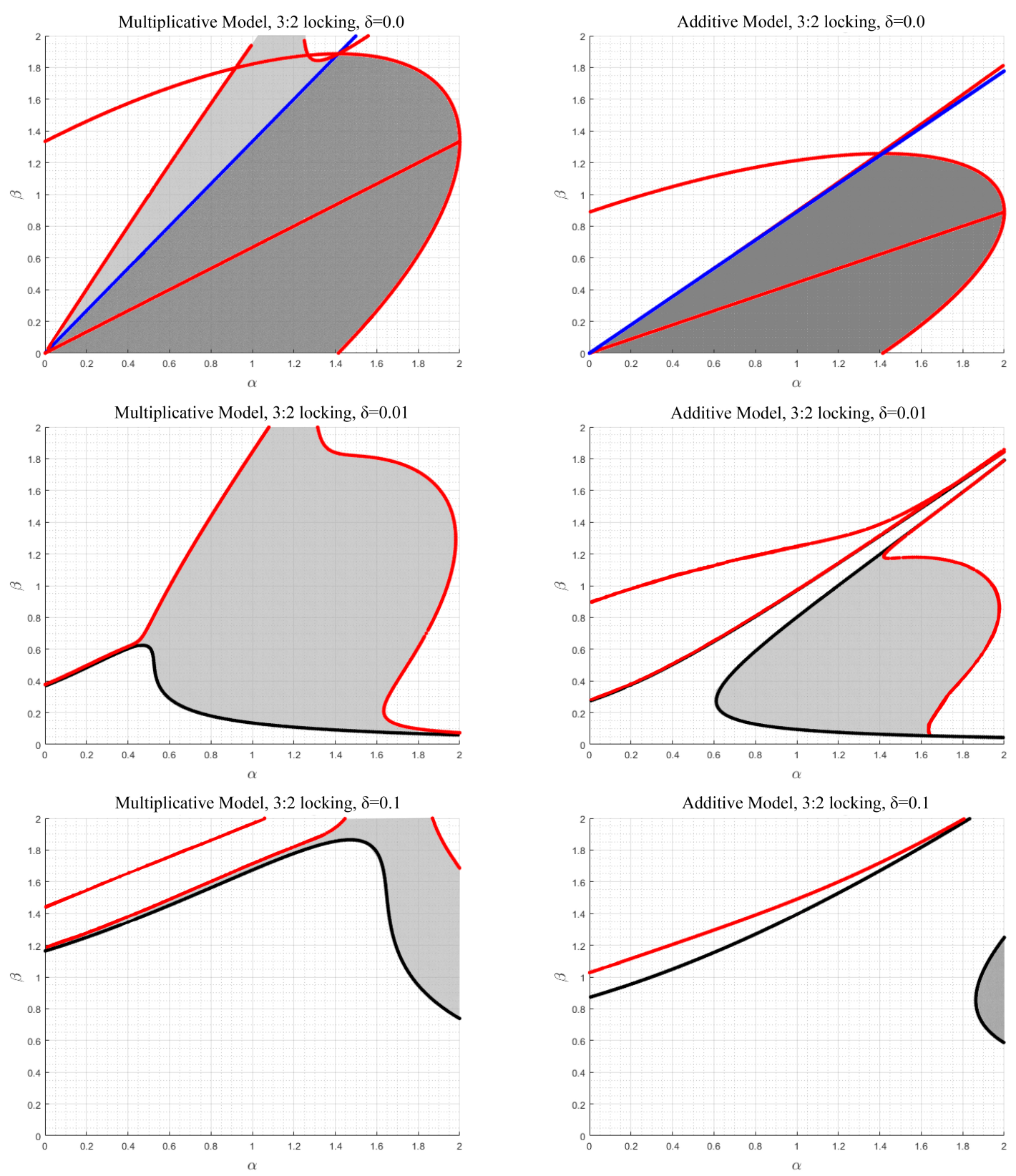

Figure 7: Multi-parameter ( $\beta$ v. $\alpha$ ) plots showing the stability and existence boundaries of $3: 2$ locking in the multiplicative and additive models as curves in $(\beta, \alpha)$ space. Red curves represent Hopf bifurcations, black curves represent limit points, and blue curves are branch points. Shaded regions represent parameter sets which have stable fixed points. The darker shade of regions in the $\delta=0.0$ case represents that these are regions which were identified analytically as well as numerically. 
Note also, that for the multiplicative model when $\delta=0.01$ and $\alpha=0.8$, varying $\beta$ from 0 to 2 gives the same limit point and Hopf bifurcation found in the one parameter plot in Fig. 4. This further verifies the numerical results found here. 


\subsection{2:3 Locking - xppaut AUTO}

2:3 locking is uniquely different than all the prior cases for two reasons. First, steady states of 2:3 locking appear as oscillations between a set of three fixed points, with a total of six fixed points when a given parameter allows for the existence of fixed points of 2:3 locking. The more important unique nature of 2:3 locking appears, however, in the fact that, even in the $\delta=0.0$ case, not all parameter choices feature fixed points of 2:3 locking. Further, no fixed point values are parameter independent, so we rely solely on numerical methods to determine the stability of this type of locking for all $\delta$ cases of the multiplicative (2.1) and additive 2.2 models.

Fig. 8 shows the numerically determined boundaries of existence (limit point curves) and stability (Hopf bifurcation curves). An important feature of the oscillator models is clear here for the $\delta=0.0$ and $\delta=0.01$ cases: in order to achieve a steady state of 2:3 locking, one or both of the coupling parameters must be 'large' (large as compared to 0.5 for example). In addition, the regions of parameters for which stable 2:3 locking states exist are smaller in comparison to those found for 1:1 or 1:2 locking. In addition, increasing $\delta$ further shrinks the stable parameter regions, and these regions occur at larger $\beta$ and/or $\alpha$ than the regions

for smaller $\delta$. This last aspect is especially clear from the fact that no stable fixed points of 2:3 locking exist for the additive model when $\delta=0.1$ (as denoted by the absence of the plot, as it would show nothing). Even for the multiplicative model, when $\delta=0.1$ the parameter region for steady states is incredibly small, requiring $\beta$ to exceed 1.7 for all applicable $\alpha$.

Note also, that for the additive model when $\delta=0.01$ and $\beta=1.2$, varying $\alpha$ from 1 to 2 gives the same limit point and Hopf bifurcation found in the one parameter plot in Fig. 4. (Note that the fixed points in the $\alpha<1$ region are excluded, because this is a different fixed point pair than the one tracked in Fig. (4). This further verifies the numerical results found here. 

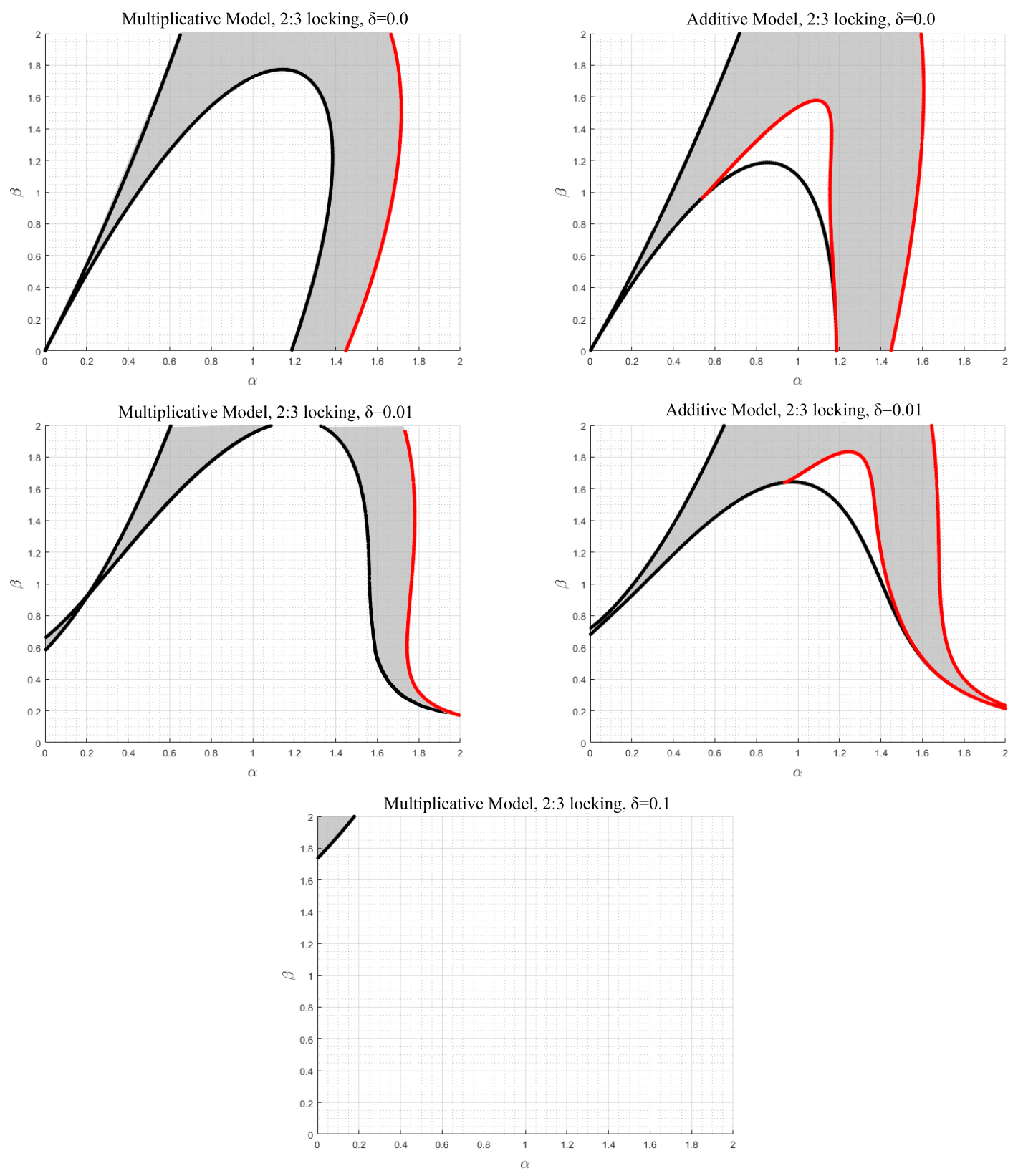

Figure 8: Multi-parameter ( $\beta$ v. $\alpha$ ) plots showing the stability and existence boundaries of $3: 2$ locking in the multiplicative and additive models as curves in $(\beta, \alpha)$ space. Red curves represent Hopf bifurcations and black curves represent limit points. Shaded regions represent parameter sets which have stable fixed points. 


\subsection{Multi-locking parameter regions}

The analytical and numerical results can be summarized by Fig. 9, which shows colored parameter regions indicating different types of stable N:M locking. Here, 1:1 locking is shown in light purple, 2:1 locking is shown in red, 1:2 locking is shown in green, 3:2 locking is shown in blue, and 2:3 locking is shown in yellow.

While it is not unexpected that an oscillator with no preferred period $(\delta=0.0)$ may have large regions of multi-locking (particularly for $\alpha \in(1.2,1.6)$ ), it is significant that even for $\delta=0.01$ (increasing the preferred period elasticity term), there are large regions in which four of the five kinds of N:M locking exist with stable steady states. Moreover, these regions have fairly acceptably small $\beta$ values, with $\alpha \in(0.6,1.6)$. As $\delta$ increases to 0.1 , however, few such regions of multi-locking remain, and only for large $\alpha$ and $\beta$. It is significant, however, that even in the $\delta=0.1$ case, the multiplicative model does still have parameter regimes with four states of stable N:M locking, regardless of the large parameter $\alpha, \beta$ sizes. A comparison to consider as a result of Fig. 9 is that, for a given $\delta$, there are larger regions of multilocking for the multiplicative model than the additive model. This seems to suggest that the description given by the multiplicative model is more ideal if complex entrainment is desired, though such a statement would require knowledge of the basins of attraction of these steady states.

It remains to determine what the basins of attraction look like, as well as their properties,

for various $\alpha$ and $\beta$ combinations, particularly those within multi-locking regions. The basins of attraction will give us an understanding of how typical it is for the oscillator models to reach a particular final steady state from an arbitrary initial condition. 

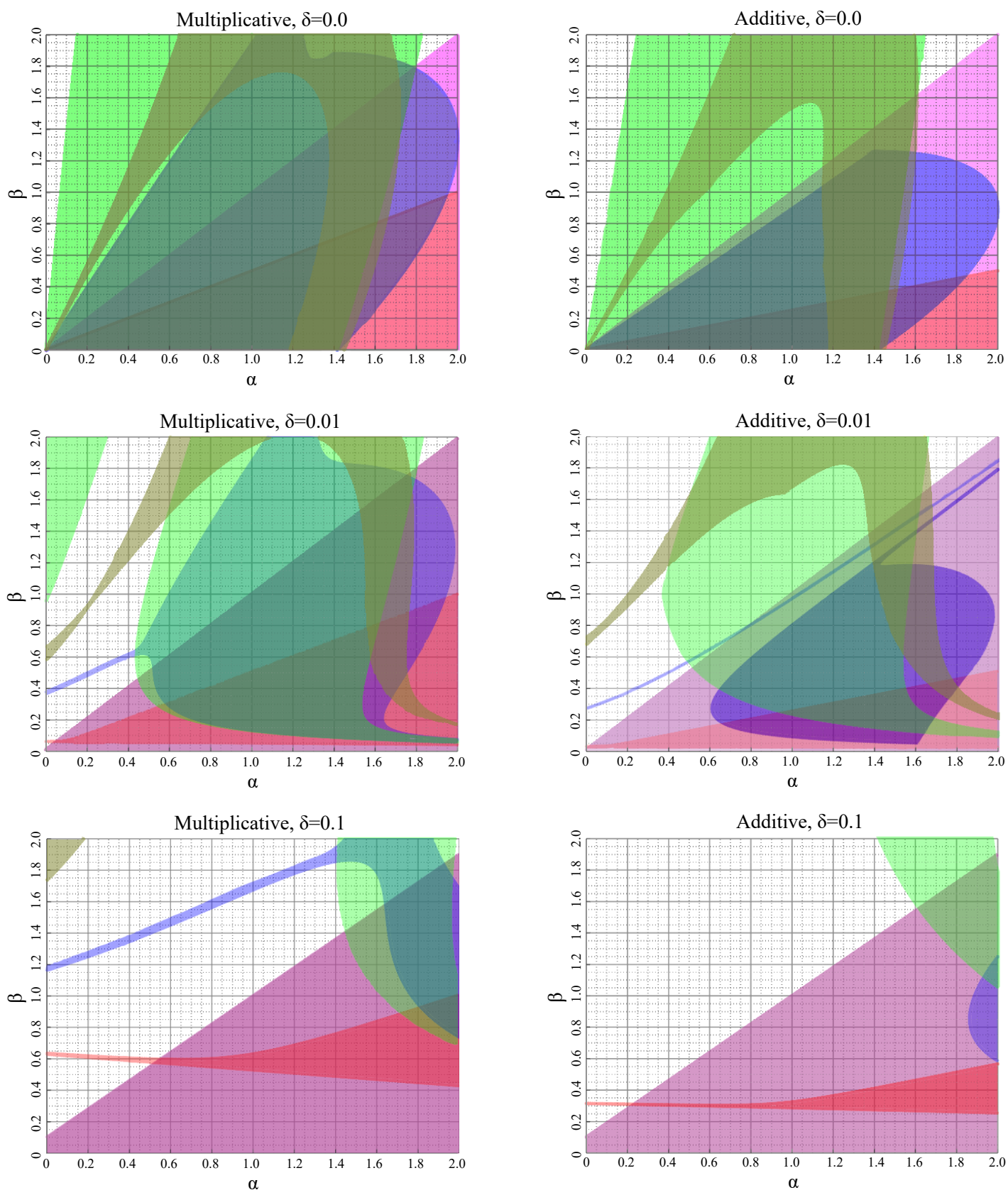

Figure 9: Parameter $(\alpha, \beta)$ regions for which the multiplicative and additive models exhibit stable fixed points of N:M locking. In these plots, green is 1:2 locking, yellow is 2:3 locking, purple is 1:1 locking, blue is 3:2 locking, and red is 2:1 locking. 


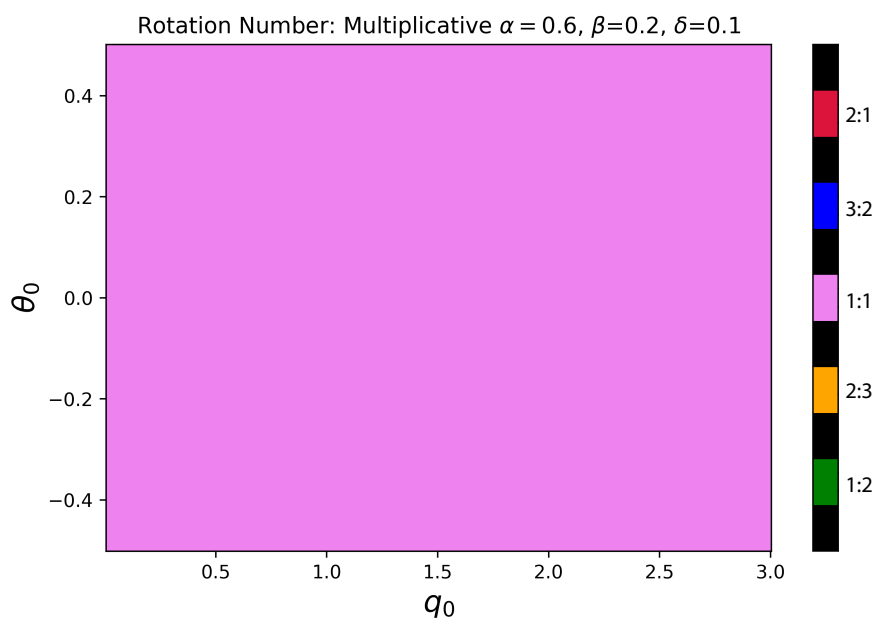

Figure 10: Final rotation number for the multiplicative model for initial conditions such that $\theta_{0} \in(-0.5,0.5)$ and $q_{0} \in(0.01,3.0)$. The entire space is dominated by convergence to the 1:1 locking state.
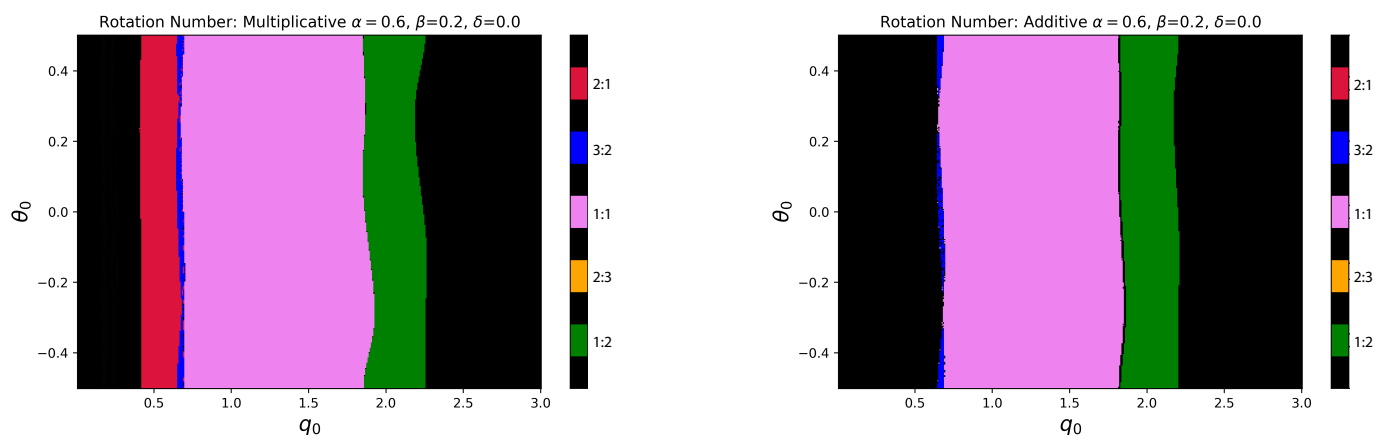

Figure 11: Final rotation number for the initial conditions such that $\theta_{0} \in(-0.5,0.5)$ and $q_{0} \in(0.01,3.0)$. Here, we see states of $1: 2,1: 1,3: 2$, and 2:1 locking for the multiplicative model (left) and 1:2, 1:1, and 3:2 locking for the additive model (right). 


\subsection{Basins of Attraction}

Recall that Fig. 9 shows the $(\alpha, \beta)$ parameter regions for which stable N:M locking states $(1: 1,1: 2,2: 1,2: 3,3: 2)$ exist (for a given $\delta$ and a forcing period $\widehat{P}=1$ ). While these models can exhibit other types of locking, we do not expect $\mathrm{N}$ :M locking with $N \geq 3, M \geq 3$ to appear until larger $\alpha$ and $\beta$ combinations, due to the qualities of the 2:3 locking parameter regions in Fig. 9. The final states of the multiplicative 2.1) and additive (2.2) oscillator

models, however, not only depend on the parameters $\alpha, \beta, \delta$, and $\widehat{P}$, but also the initial conditions $\left(\theta_{0}, q_{0}\right)$. Given an initial $\left(\theta_{0}, q_{0}\right)$ and $n$ many iterations, the model may converge to a state of N:M locking, a quasi-periodic state, some periodic orbit, or not converge. We can characterize the final state by the rotation number $\lim _{n \rightarrow \infty} \theta_{n} / n$, when $\theta_{n}$ is allowed to run free (eliminating $\left.\bmod { }_{[-0.5,0.5]} 1\right)$. If the oscillator is in a state of $\mathrm{N}$ :M locking after sufficient iterations, the rotation number for the given initial conditions should be approximately $N / M$. However, if the model is in a quasi-periodic state, it may appear to have a rotation number $N / M$ despite not being a stable periodic state of $\mathrm{N}: \mathrm{M}$ locking. Thus, it is necessary to also check the periodicity of final states. For all the figures depicting rotation numbers (Figs. 10 . 18), a 400x400 grid of initial condition points is utilized, with $n=4000$ iterations. Each IC point $\left(\theta_{0}, q_{0}\right)$ is then colored according to whether the final state is periodic and its rotation number $\left(\theta_{4000} / 4000\right)$ falls within the desired N:M locking regions. Periodicity is determined by whether at least two points $\left(\theta_{n}, q_{n}\right)$ of the final ten iterations being equal within $10^{-10}$. If the rotation number $r \in[0.45,0.55]$, the point is marked as $1: 2$ locking. If $r \in[0.6,0.7]$, the point is marked as 2:3 locking. If $r \in[0.95,1.05]$, the point is marked as 1:1 locking. If $r \in[1.45,1.55]$, the point is marked as 3:2 locking. Finally, if $r \in[1.95,2.05]$, the point is marked as 2:1 locking. Changing this tolerance of $\approx 0.05$ slightly does not significantly impact the overall shapes of the basins of attraction for all shown figures (Figs. 10 . 18).

First, consider a region where only one type of locking has stable steady states: the multiplicative oscillator model for when $\delta=0.1, \alpha=0.6$, and $\beta=0.2$. Here, of the types of locking investigated, only 1:1 locking fixed points are limiting points of trajectories. As such, it is expected that the domain of attraction of this 1:1 fixed point should be large-i.e., 
when starting at various initial points $\left(\theta_{0}, q_{0}\right)$, after sufficient time the trajectory should converge to a point $(\theta, q)$ close to $(0,1)$ (the exact value of this fixed point is given in section 3.1, equation (3.4)). Iterating the model at various initial conditions $\theta_{0} \in(-0.5,0.5)$ and $q_{0} \in(0.01,3)$, Fig. 10 shows that, in fact, the domain of attraction is the whole $400 \times 400$ point space. This is exactly what we expect for a parameter set in which only 1:1 locking is stable (though higher levels of locking may be exhibited for larger $q$, such as for example 1:5 locking, where $q \approx 5$, out of the range of this figure).

In regions of multi-locking, it becomes increasingly important to determine the domains of attraction for fixed points of $1: 1,1: 2,2: 1,2: 3$, and 3:2 locking. When at a fixed point of N:M locking, the scaled period of an oscillator model should be $q \approx M / N$ (as opposed to the rotation number, which is $\approx N / M)$. As such, in regions of multi-locking, we expect limits of trajectories corresponding to N:M locking should occur in a neighborhood around $q_{0}=M / N$. For example, consider the multiplicative model (2.1) and additive model (2.2) when $\alpha=0.6$ and $\beta=0.2$ with $\delta=0.0$. Fig. 9 indicates that the multiplicative model should exhibit stable 1:1, 2:1, 1:2, and 3:2 locking and the additive model should exhibit stable 1:1, 1:2, and 3:2 locking. However, without looking at the domains of attraction, it is unclear exactly what initial conditions will result in such final states. Iterating at various initial conditions $\left(\theta_{0}, q_{0}\right)$, Fig. 11 shows that in the approximate range $q_{0} \in(0.4,2.3)$, the multiplicative and additive oscillator models converge to exactly the states indicated by Fig. 9. Around $q_{0}=0.5$, the multiplicative model converges to 2:1 locking. In a small region near $q_{0}=0.66$, the multiplicative model converges to 3:2 locking. The largest domain of attraction is 1:1 locking, which is not centered at $q_{0}=1$, but includes it. Finally, near $q_{0}=2.0$, the multiplicative model converges to 1:2 locking. Similar regions occur for the additive model, with the domain of attraction of 1:1 locking being dominant and of 3:2 being the smallest. Therefore, as expected, the domains of attraction for stable N:M locking states occur in neighborhoods around $q_{0} \approx M / N$. However, what we could not predict were how large these regions would be. Though it is not surprising that 1:1 locking may be dominant in both models, the domain of attraction for 3:2 locking is incredibly small, making it a state that cannot be reached by the models unless the oscillator already begins close to this state in terms of its initial period $q_{0}$. 
In the above case (Fig. 11), the domains of attraction appear connected, continuous, and fairly smooth on their boundaries. Consider instead the case where $\alpha=1.75, \beta=0.4$ (as such, $\alpha$ is large, and $\beta$ is small). Further, vary $\delta$ to be $0.0,0.01,0.1$. Fig. 12 shows the domains of attraction for these various cases, with the multiplicative model in the left column, the additive model in the right column, and $\delta$ increasing with each subsequent row. The first visual difference with Fig. 11 is that the domains of attraction have boundaries which are dependent both on $q_{0}$ as well as $\theta_{0}$. Meaning, consider for example the 1:1 locking domain of attraction of the additive model with $\delta=0.01$ (pink region in the bottom-right plot in Fig. 12. Some $\theta_{0}$ have a larger maximum $q_{0}$ for which the system converges to its 1:1 fixed point (for example, $\theta_{0}=-0.2$ has a larger $\max \left(q_{0}\right)$ than $\theta_{0}=0.2$ ). Another difference in the characteristics of the domains of attraction is that for a fixed $\theta_{0}$, the domain is discontinuous-other types of N:M locking fracture the domain, either at the border or even within. For example, for the additive model when $\delta=0.01$ (bottom-right plot in Fig. 12), the domain of attraction of 2:3 locking can be found what looks to be "within" the domain of attraction of 1:1 locking.

It is difficult, however, from the given figures to determine whether the regions themselves are connected, due to the mesh grid being of a limited size. To investigate this, take the case of the additive model when $\alpha=1.75, \beta=0.4$, and $\delta=0.01$ as it was shown in Fig. 12. Let us vary $q_{0}$, instead, from 0.01 to 0.5 in 400 evenly spaced points, "zooming in" on the region of 2:1 locking which, due to low resolution, looks disconnected in Fig. 12. Increasing the resolution, the top plot in Fig. 13 indicates that the additive model exhibits fractal behavior at the boundaries of domains of attraction. Moreover, extending $\theta_{0}$ periodically suggests that the domain of attraction may be connected, though it would take a plot of incredibly high resolution (compared to 400x400) to investigate this. Magnifying the highlighted region (white border), which encloses initial conditions $q_{0} \in(0.2,0.23)$ and $\theta_{0} \in(-0.2,-0.17)$, the right top plot of Fig. 13 shows that the region continues to appear fractal. Notice also that there are points throughout the region which are associated with rotation numbers of 3:2 and 1:1 locking. Finally, we can magnify once more on the region where $q_{0} \in(0.210,0.215)$ and $\theta \in(-0.186,-0.181)$, seen in the bottom plot of Fig. 13 , to see that the fractal appearance continues. One aspect of the bottom plot of Fig. 13 which is not clear is what the black 

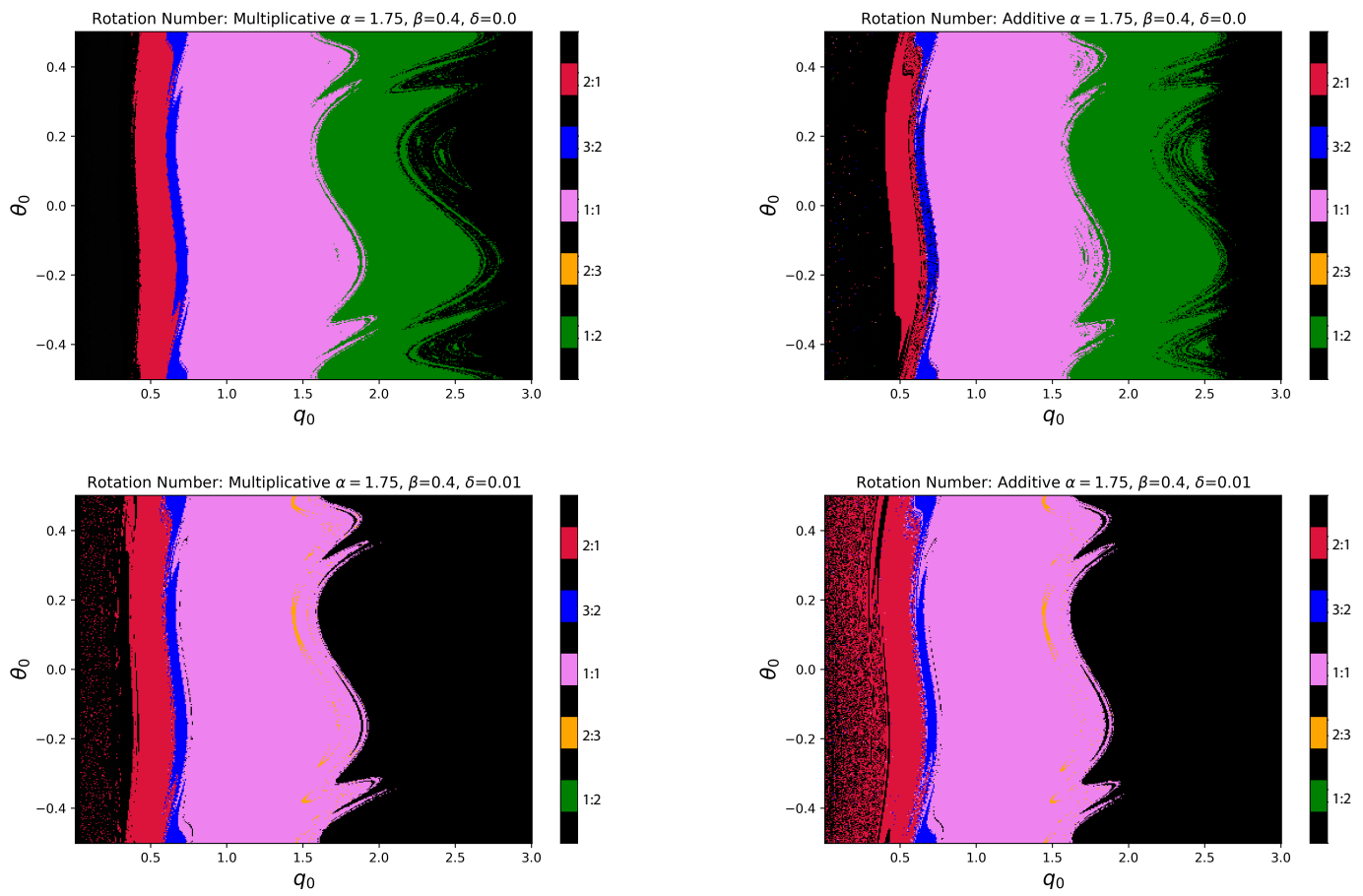

Figure 12: Final rotation number when varying the initial conditions such that $\theta_{0} \in$ $(-0.5,0.5)$ and $q_{0} \in(0.01,3.0)$. Though many of the rotation number regions, corresponding to basins of attraction, were predicted by Fig, 9 , there are four regions that were unexpected. For the multiplicative model, $\delta=0.0$, rotation numbers corresponding to 3:2 locking (blue) and 1:2 locking (green) were not predicted; upon investigation, these regions correspond to 6:4 locking and 2:4 locking. For the additive model, $\delta=0.0$, rotation numbers corresponding to 1:2 locking (green) were not predicted; upon investigation, this region is 2:4 locking. And, finally, for the multiplicative model, $\delta=0.01$, rotation numbers corresponding to 3:2 locking (blue) were not predicted; this region corresponds to 6:4 locking. 
colored regions mean in this particular plot-in particular, whether these regions represent a type of locking not investigated, or if those parameter sets are not periodic in their final state. Upon investigation, we find that, particularly within the bulls-eye portion of the bottom plot of Fig. 13, much of the region which does not end in 2:1 locking ends in a state of $5: 2$ locking (with a rotation number in the range [2.45, 2.55]), as seen in Fig. 14 .

In other cases, the connectivity of the domains of attraction appear more obvious. Consider the parameter set $\alpha=0.8, \beta=0.35$, and $\delta=0.01$ for both the additive and multiplicative models: Fig. 15 shows the domains of attraction for these cases. Focusing on 1:1 and 2:3 locking, keeping periodicity of $\theta$ in mind, the domains appear connected in Fig. 15 , though for a fixed $\theta_{0}$ or $q_{0}$, there are discontinuities, such that when varying $q_{0}$ linearly, the final state oscillates between 1:1 and 1:2 locking.

In Fig. 12, Fig. 13, and Fig. 15, the domains of attraction appear fractal in nature, which can particularly be seen in Fig. 12 but is also evident in the 2:1 and 3:2 locking regions of Fig. 15. This fractal behavior is particularly apparent for the additive model when $\alpha=0.8, \beta=1.9$, which features $1: 2$ and $2: 3$ stable locking in the $\delta=0.0$ and $\delta=0.01$ cases. Fig. 16 shows how, though some portions of the domains of attraction are continuous and connected, there is a great deal of fractal behavior, particularly for 2:3 locking in the $\delta=0.01$ case. In addition, on the right hand side, extending $\theta$ periodically would once again show a connectivity of the regions of $1: 2$ and 2:3 locking (though, once again, this does not say that such regions are generally connected, especially considering the fractal behavior on the left hand side).

So far, the only cases we have looked at are where at least one of $\alpha$ or $\beta$ is small (as in, $<1$ ). Fig. 11, in comparison with later figures, seems to suggest that when $\alpha$ or $\beta$ is large, the domains of attraction become increasingly 'distorted' in shape and fractured to exhibit fractal behavior. This is supported by Fig. 17, which shows the multiplicative and additive models in the case of $\alpha=0.9, \beta=0.2$, and $\delta=0.0,0.01$. Here, there is minimal fractal behavior (primarily seen in the 2:1 case), and the domains of attraction seem to primarily depend upon $q_{0}$ rather than $\theta_{0}$ otherwise. However, to contrast, consider the case when $\alpha$ and $\beta$ are both large $(>1)$; in particular, let $\alpha=1.8, \beta=1.48$, and $\delta=0.0,0.01,0.1$. Fig. 18 shows how all of the domains of attraction of stable N:M locking are incredibly distorted 

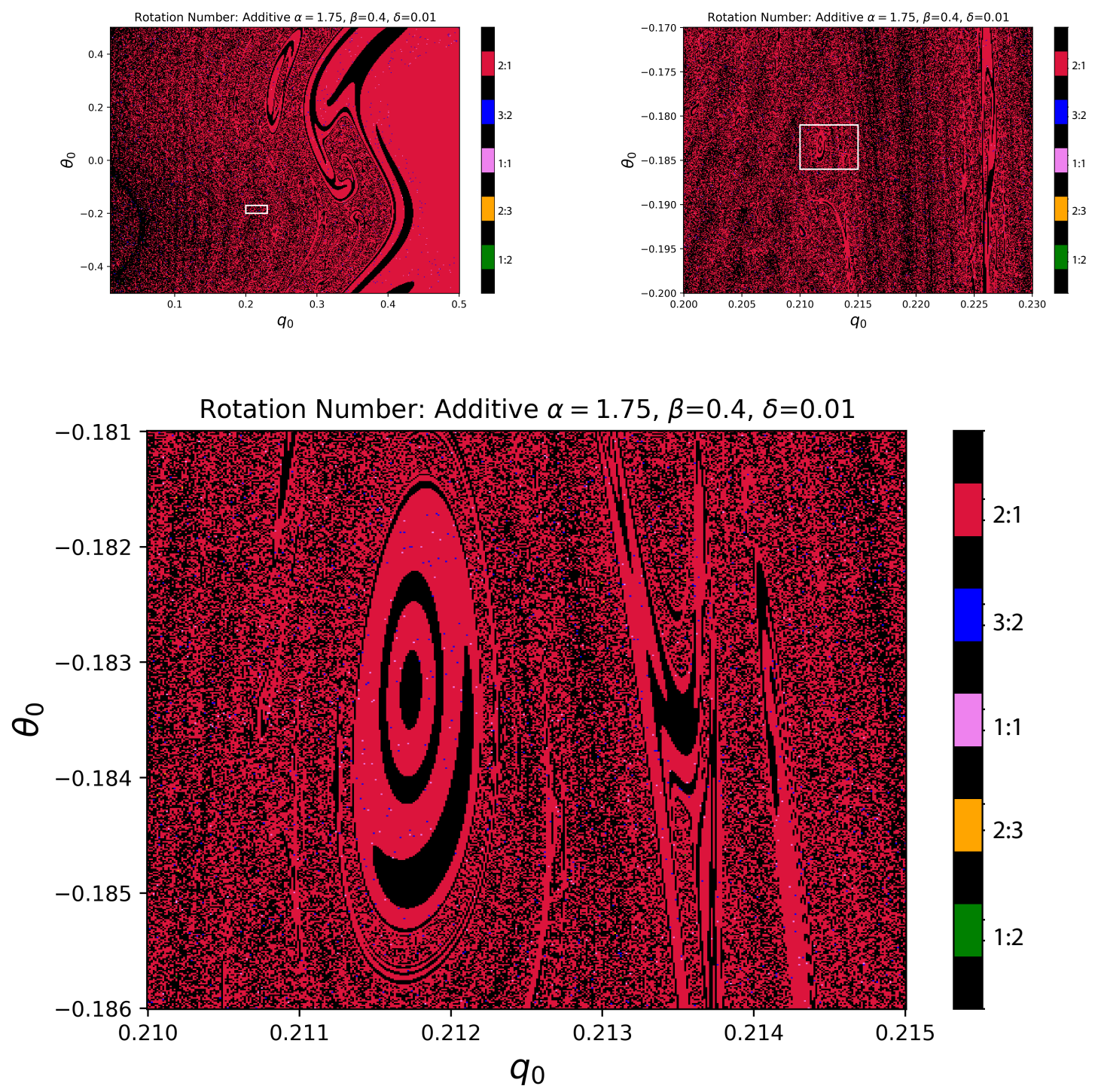

Figure 13: Final rotation number for various initial condition $\left(\theta_{0}, q_{0}\right)$ ranges. Compared to Fig. 12, the plot on the top shows a higher resolution image for initial conditions $q_{0} \in$ $(0.01,0.5)$ and $\theta_{0} \in(-0.5,0.5)$. Similarly, the plot on the bottom shows a higher resolution image for initial conditions $q_{0} \in(0.2,0.23)$ and $\theta_{0} \in(-0.2,-0.17)$. White boxes highlight the regions being magnified 


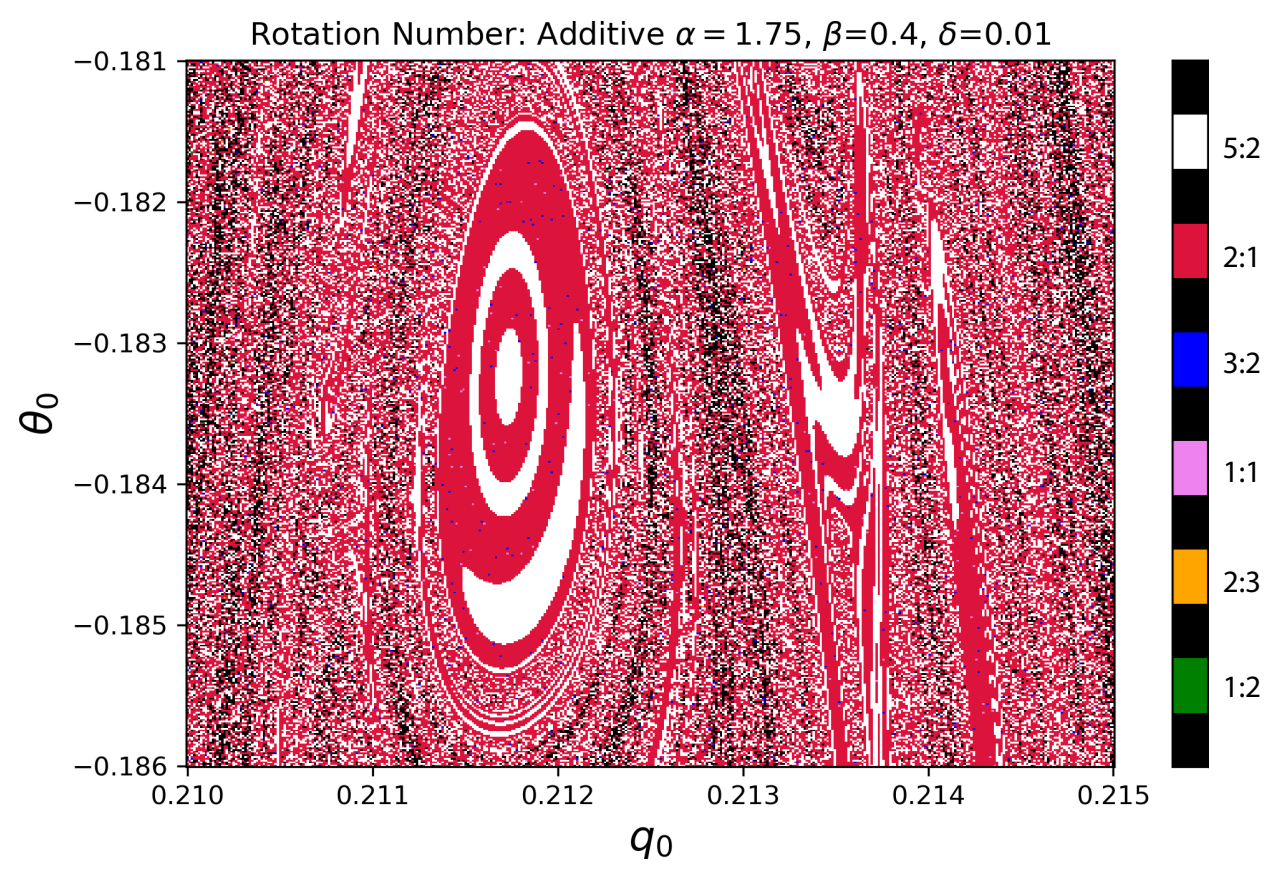

Figure 14: Final rotation number for various initial condition $\left(\theta_{0}, q_{0}\right)$ with $\theta_{0} \in$ $[-0.186,-0.181]$ and $q_{0} \in[0.210,0.215]$. The plot is identical to Fig. 13 , except that an additional white region is colored for initial conditions whose final states are 5:2 locking.
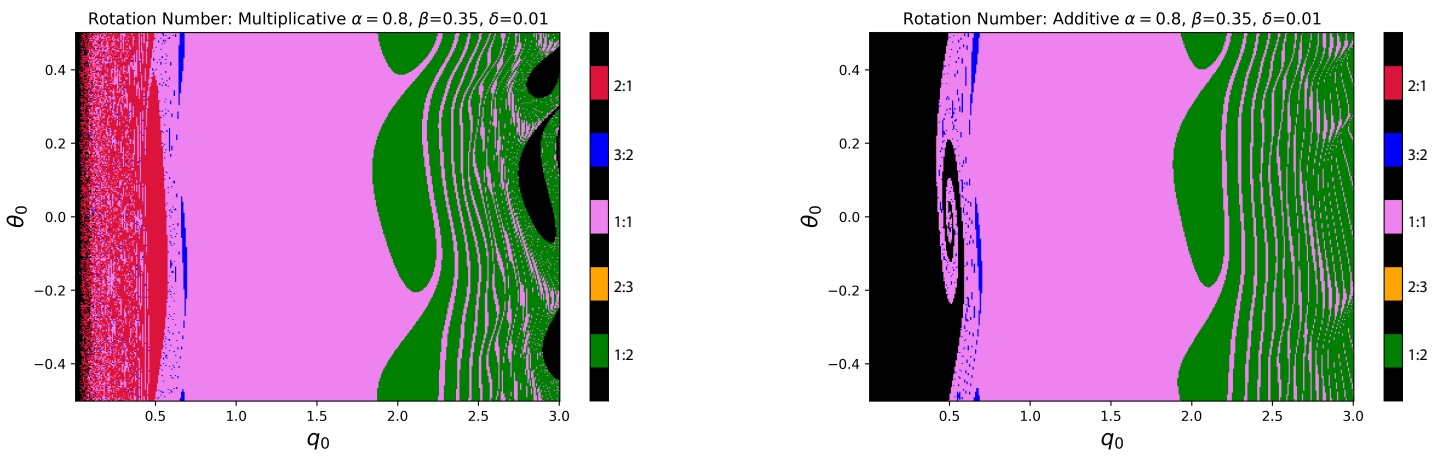

Figure 15: Final rotation number for initial conditions such that $\theta_{0} \in(-0.5,0.5)$ and $q_{0} \in$ $(0.01,3.0)$ for the multiplicative (left) and additive (right) models. 


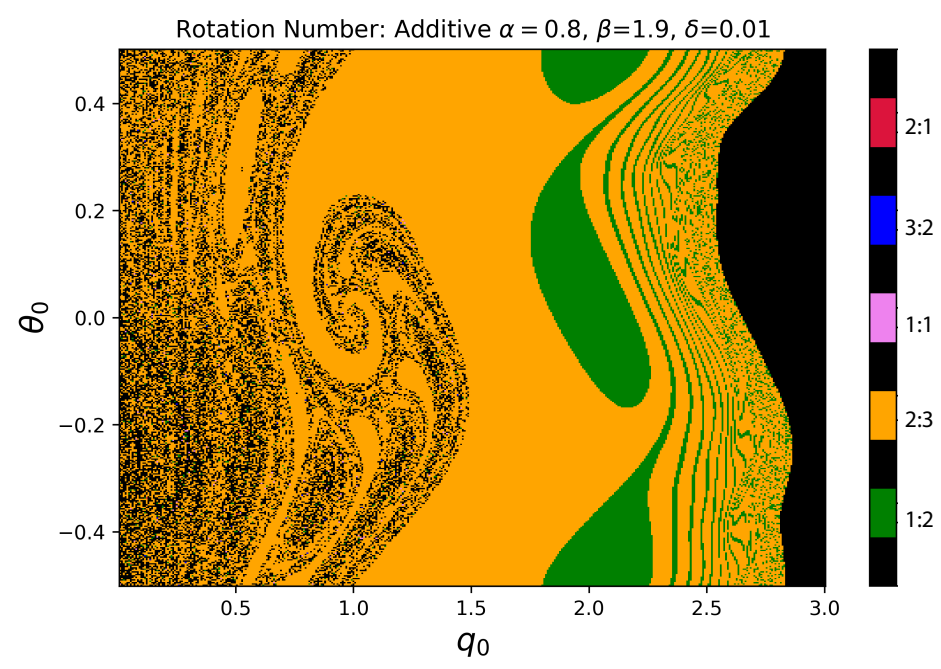

Figure 16: Final rotation number when varying the initial conditions such that $\theta_{0} \in$ $(-0.5,0.5)$ and $q_{0} \in(0.01,3.0)$.

in shape (as compared to Fig. 17) and feature fractal behavior at either the boundaries or throughout large sections.

Fig. 12 and Fig. 18 have phase coupling terms $\alpha$ close to one another; there are also two additional similarities between these cases beyond the value of $\alpha$. Both of these cases feature domains of attraction which were not predicted by Fig. 9-meaning that there is a periodic state with a rotation number that corresponds to $N: M$ locking but may not in fact be a state of N:M locking. In Fig. 12, the multiplicative model for $\delta=0.0$ exhibits what appears to be 3:2 (blue) and 1:2 (green) locking final stable states-however, when choosing a test point within the regions, it becomes evident that these are in fact domains of attraction for states of 6:4 and 2:4 locking. We know this because the final periodic state features a set of 4, not 2, fixed points. Since the rotation number only depends on the fraction of $N$ to $M$, 6:4 and 3:2 locking will appear identical in such plots intended to illuminate the basins of attraction. In addition, through this same method of test points, we find that Fig. 12 shows the domain of attraction for 2:4 locking for the additive model $(\delta=0.0)$ and the domain 
of attraction for 6:4 locking for the multiplicative model $(\delta=0.01)$. For the parameter set $\alpha=1.8, \beta=1.48$, several unexpected regions corresponding to $1: 2$ were found, in fact found to be either period doubling case (2:4) or quadrupling case (4:8). Generally, these unexpected regions which are domains of attraction of $\frac{a N}{a M}, a$ an integer, are found for cases of large $\alpha$, as in Fig. 12 and Fig. 18 . 

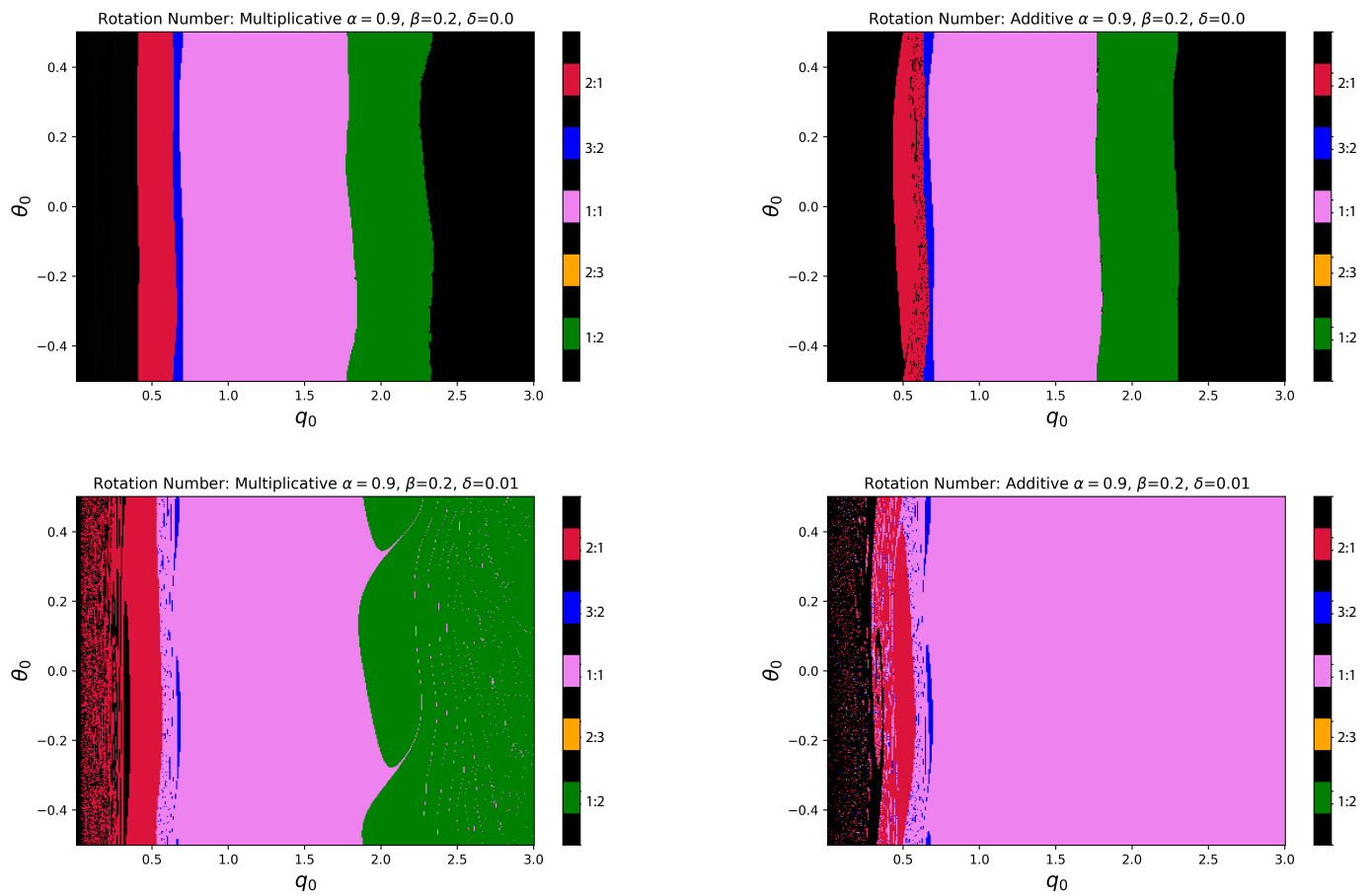

Figure 17: Final rotation number when varying initial conditions such that $\theta_{0} \in(-0.5,0.5)$ and $q_{0} \in(0.01,3.0)$ for the multiplicative (left) and additive (right) oscillator models. 

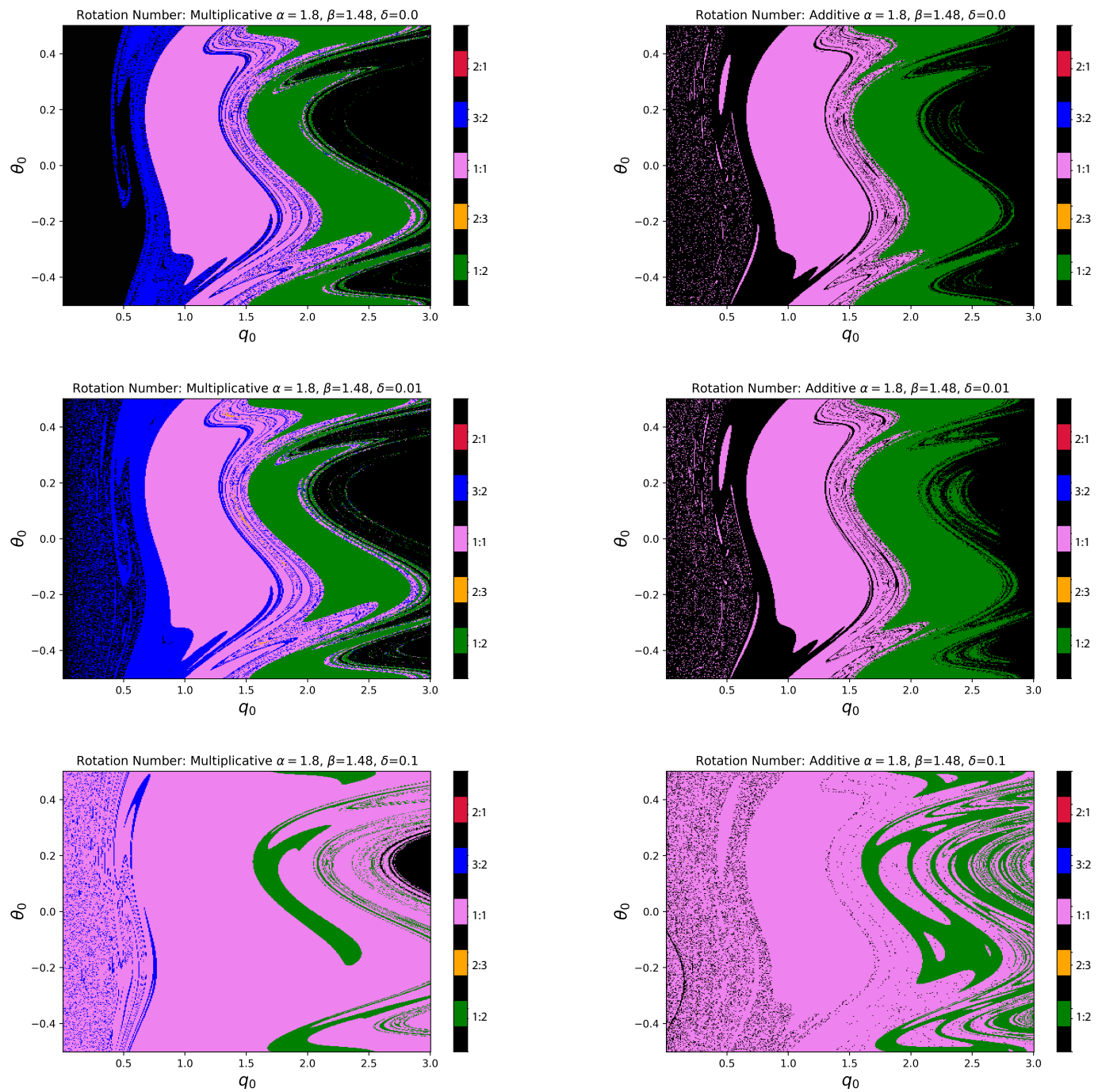

Figure 18: Final rotation number when varying initial conditions such that $\theta_{0} \in(-0.5,0.5)$ $q_{0} \in(0.01,3.0)$. Though many of the rotation number regions, corresponding to basins of attraction, were predicted by Fig, 9 , there were four unexpected regions. For the multiplicative and additive models, $\delta=0.0$ and $=0.01$, rotation numbers corresponding to 1-2 locking (green) were not expected. Upon investigation, the multiplicative cases correspond to 2:4 locking, the additive case with $\delta=0.0$ corresponds to $2: 4$ locking, and the additive case with $\delta=0.01$ corresponds to 4:8 locking. 


\subsection{Discussion}

Recall that more recent oscillator models of synchronization include period adaptation terms (unlike the standard model (1.1)) in order to account for an oscillator's ability to change its period and maintain the beat after a stimulus is discontinued. This added dimension in period or frequency can include a period coupling term and/or preferred period elasticity. However, there are also models of entrainment or beat-keeping that are not centered around the idea of an oscillator (or error correction). The various models, though at times designed to understand different systems, have similar overarching themes and properties.

Recall that Mosheiff et al. described the entrainment of a cell cycle $T_{n}$ to the cellular circadian clock $T_{\text {osc }}$ by a second order phase equation (1.4):

$$
\theta_{n+1}=\theta_{n}+\frac{\tau_{0}}{T_{o s c}}(1-\gamma)+\gamma\left(\theta_{n}-\theta_{n-1}\right)+\tilde{k} \sin \left(2 \pi \theta_{n}\right)
$$

Here, $\tau_{0}$ is the intrinsic cell-cycle period, $k$ is the coupling between the cell cycle and circadian

oscillator, and $\gamma$ defines mother-daughter (cell) coupling. Phase is given by $\theta_{n}=\frac{t_{n}}{T_{o s c}}$ where $t_{n}$ is the birth time of a cell (see section 1.0.2). The nonlinearity of the model was provided a mechanism for an inequality observed in experiments [22].

It is not immediately apparent perhaps how this second order phase equation relates to either the multiplicative or additive models described here. However, we can generate an analogous description of phase $\theta_{n+1}=f\left(\theta_{n}, \theta_{n-1}\right)$ for the multiplicative and additive models by introducing small perturbations and rearranging terms to eliminate the period component $q_{n+1}$. When looking at the Mosheiff et al. model (1.4), the only apparent coupling term to the circadian oscillator (forcing stimulus) is $\tilde{k}=k / T_{\text {osc }}$ : the forcing amplitude of the cellular circadian clock. As such, we set $\beta=0$ in the additive and multiplicative models, making them equivalent, and determine the following second order equation for phase:

$$
\theta_{n+1}=\theta_{n}+\delta \widehat{P}+(1-\delta)\left(\theta_{n}-\theta_{n-1}\right)-\frac{\alpha \delta}{2 \pi} \sin \left(2 \pi \theta_{n}\right)
$$

Comparing (1.4) and our models (10.1) directly, we can identify how parameters of the Mosheiff et al. model (mother-daughter coupling term $\gamma$, cell cycle to circadian clock coupling 
$k$, intrinsic cell-cycle period $\tau_{0}$, and forcing period $T_{\text {osc }}$ ) are analogous to parameters of the additive and multiplicative models (phase coupling term $\alpha$, preferred period elasticity $\delta$, scaled forcing period $\left.\widehat{P}=I O I_{n} / \widetilde{P}\right)$ :

$$
\left\{\begin{array}{l}
1-\gamma \sim \delta \\
\frac{k}{T_{\text {osc }}} \sim-\frac{\alpha \delta}{2 \pi} \\
I O I_{n} / \widetilde{P} \sim \tau_{0} / T_{o s c}
\end{array}\right.
$$

As such, $1-\gamma$, where $\gamma$ is mother-daughter coupling, relates to the preferred period elasticity term. Since an inverted relationship is used for relating the intrinsic period and forcing period (3rd equivalence relation in $(10.2)$ ), a larger $\gamma$ results in the model being more resistant to entraining to the forcing period $T_{\text {osc }}$. In addition, the relations in 10.2 draw a comparison between coupling terms with the stimulus: the cell cycle-circadian clock coupling $k$ is proportional to the the multiplicative/additive phase coupling term $\alpha$ (but also the preferred period elasticity $\delta$ ). It is not quite so important how exactly the parameters translate, but there is sufficient similarity to directly compare the results and implications of both models.

In terms of properties and results, there are several similarities between the multiplicative/additive models and the Mosheiff et al. kicked cell cycle model, beyond just their nonlinearity and order. First, both models exhibit periodic and quasi-periodic regions, and, more importantly, the models both feature large parameter regions of higher dimensional locking (specifically, period $n$ (i.e., 1:n) locking [22]). However, in our models, when $\beta$ is set to zero, much of the higher dimensional $N: M$ locking is lost for nonzero $\delta$ (see Fig. 9 , This speaks to, perhaps, the lack of a direct correspondence in parameters, particularly when comparing the coupling terms between the oscillator and its stimulus. In addition, the original Mosheiff et al. model (1.4) includes noise at each $n$, and they showed that, even in the presence of high noise, some fixed points beyond 1:1 locking were retained (i.e. these features were robust to noise) [22]. It would be interesting, as such, to see how robust the multiplicative and additive models' properties are if some stochastic dynamics are added, since most biologically realistic systems feature noise in their inputs.

Oscillator models are a common dynamical systems approach to synchronization studies, such as here and in Mosheiff et al., but recall that Bose et al. utilized a neuro-mechanistic 
approach to understanding entrainment [13]. They modeled beat perception by considering dynamics which originate from the behavior of a "beat generator neuron" (BG), modeled by a leaky integrate and fire model. The BG learns the period and timing of the stimulus through an internal parameter $I_{\text {bias }}$ that regulates the BG's excitability. Two learning rules, a period learning rule $L R_{T}$ and a phase learning rule $L R_{\phi}$, affect $I_{\text {bias }}$ in order to "teach" the sequence to the BG. Simulations showed that both rules are necessary for entrainment with zero phase to occur, which is what we would also expect of the multiplicative and additive models. However, what makes Bose et al.'s model unique in comparison with the models used here is that the stimulus-model coupling is both nonlinear and non-periodic. In particular, the period of the BG leaky integrate and fire model is given by:

$$
T=\tau \log _{e}\left(\frac{I_{\text {bias }}}{I_{\text {bias }}-1}\right)
$$

where $\tau$ is the membrane time constant. Most entrainment models utilize coupling terms that are periodic, in contrast with Bose et al. [13]. The multiplicative and additive models, the Saigusa et al. model (1.3), and also the Mosheiff et al. model (1.4) all feature sinusoidal coupling terms. Moreover, the non-periodic nature of the dynamics of the BG neuron mean that it will never converge to a periodic orbit when receiving stationary stimuli $\left(I O I_{n}=T^{*}\right.$, corresponding to the case where $\left.\widehat{P}_{n}=\widehat{P}\right)$, quite unlike the models presented here (2.1), (2.2). The discrete nature of the learning rules $L R_{\phi}$ and $L R_{T}$ means that the final rhythm of the oscillator upon "synchronization" is not exact.

Despite the neuromechanistic model and oscillator models operating other fairly different mechanisms, there are some properties which are exhibited by both. Period memorization and synchronization-continuation are two properties common in biological oscillators, such as the $P$. polycephalum mentioned below. The standard oscillator model (1.1), operating individually (not in a network), has neither property, as it has no mechanism for maintaining the stimulus period and will revert to some natural period $\widehat{T}$ if the stimulus is discontinued. Conversely, the multiplicative and additive models, when there is no preferred period $\delta=0.0$, learn the stimulus period and are able to maintain it after the stimulus is discontinued. The Bose et al. model also exhibits this ability to learn and maintain a new frequency [13]. 
Additionally, both the Bose et al. model and, at the very least, the Loehr et al. (multiplicative, $\delta=0.0$ ) models both exhibit asymmetry in response to tempo conditions. Loehr et al. conducted experiments that investigated how musicians respond to tempo changes, and found that they respond better to decreasing than increasing tempos. This asymmetry was predicted by the multiplicative oscillator model [14]. However, the Bose et al. model predicts that beat generator mechanisms adapt better to increasing tempos rather than decreasing. It is difficult to say whether the experiments conducted by Loehr et al. necessarily support the oscillator model's asymmetry, as musicians are often trained for decreasing their tempo rather than increasing [14]. The sign of the asymmetry is not as relevant, for it could be adjusted by slightly redefining intrinsic dynamics or parameters. We did not investigate the predicted asynchrony of the additive model, so this would be something to investigate in the future, particularly in comparison with the multiplicative and neuromechanistic models. Moreover, it would be valuable to determine how the asymmetry is impacted by the various parameters, especially for nonzero $\delta$.

One property, however, that the neuromechanistic model is not designed for is N:M locking states. Both the multiplicative and additive models have large parameter regions which can exhibit at least 2, at times 5 (of 5 investigated), different types of complex N:M entrainment. The neuromechanistic model is designed to learn and behave in a 1:1 state, so it does not account for biological oscillating systems' ability to perform N:M locking. However, as some such systems involve networks of oscillators (ex: neuronal oscillators), it remains to understand whether N:M locking can be achieved at the network level. For example, Large and Palmer considered a computational model which is a system of oscillators with different periods, organized in a metrical structure to entrain to some global musical rhythm stimulus [10]. Same as in the additive and multiplicative models, Large and Palmer use oscillators with flexible periods, and add a temporal receptive field which restricts the time window during which an oscillator can adjust its phase. They posited that, since musical rhythms consist of various parts at different periodic modes, that is necessary to utilize multiple oscillators at different periods to track different period components [10]. The period adaptation term utilized by Large and Palmer does not include a natural period, and is formulated like the 
multiplicative model, in that the period coupling is multiplied by the period at event $n$ :

$$
p_{n+1}=p_{n}\left(1+f\left(\theta_{n}\right)\right)
$$

where $f\left(\theta_{n}\right)$ is the period coupling term which includes a coupling strength, periodic dependence on $\theta_{n}$, an expectation parameter, and the temporal restriction.

One could construct a comparison to the work by Large and Palmer [10] by considering a network of oscillators with period descriptions which are closer to the additive and multiplicative models (2.2), 2.1) presented here. Adding a preferred period to the oscillators may help in entrainment, depending upon their distribution; for example, oscillators with intrinsic periods close to the stimulus could entrain 1:1, while others $\mathrm{N}: \mathrm{M}$ depending on the ratios of intrinsic to forcing period. In Large and Palmer's network, the hierarchical structure automatically categorizes the input events, though a looser structure and coupling scheme may permit certain oscillators to operate at points of N:M locking. In addition, it would be advantageous to understand how Large and Palmer's network behaves with an alternative period description (additive).

When the oscillators operate in a coupled network of oscillators, we expect that only specific conditions or methods of coupling would allow for steady states of N:M locking at global levels (ex: weakly coupled, coupling to only particular neighbors, etc.). This would certainly be of interest to investigate going forward, in order to draw more comparisons with more biologically motivated models of oscillating biological systems. Recent work by Kim et al. investigated N:M locking (i.e., mode locking) in a neural network of nonlinear oscillators at a range of distinct periods experiencing periodic forcing. Specifically, they utilize a gradient frequency neural network to determine the stability of fixed points of harmonic and subharmonic locking (N:1 and 1:M locking), and found that the model explains some nonlinear components to human auditory processing [26]. As such, going forward, we would like to investigate how the multiplicative (2.1) and additive (2.2) oscillator models behave in networks, with particular attention drawn the the structure and strength of coupling and the distribution and strength of intrinsic periods. 
Overall, the multiplicative and additive oscillator models share some of the universal properties of neural oscillators, so by studying their properties we improve our understanding of synchronization, particularly in the contexts of beat tracking and musical rhythm interpretation. Both feature large parameter regions of stable multi-locking, which, for those beyond 1:1, decrease in size as the preferred period elasticity $\delta$ increases, with 1:1 locking tending to dominate the initial condition ranges investigated. As the locking parameters $\alpha$ and $\beta$ increase, the basins of attraction become more 'distorted', where (for a desired final $N$ : $M$ locking state) the range of possible initial scaled periods $q_{0}$ for a chosen initial phase $\theta_{0}$ may differ wildly between the possible choices of $\theta_{0}$. For larger locking parameters, we see fractal behavior at the boundaries and at times throughout the basins of attraction. Many of the results suggest some sort of connectivity when $\theta$ is extended periodically, but this cannot be verified due to the fractal behavior previously noted. Finally, these models suggest that a stronger intrinsic period of an oscillator leads to an inability to entrain at complex intervals (N:M locking) unless the period and phase coupling terms are large. 


\section{Bibliography}

[1] M. D. C. Belle and C. O. Diekman, "Neuronal oscillations on an ultra-slow timescale: daily rhythms in electrical activity and gene expression in the mammalian master circadian clockwork," Eur. J. Neurosci., vol. 48, no. 8, pp. 2696-2717, 2018, doi: 10.1111/ejn.13856.

[2] L. M. Ward, "Synchronous neural oscillations and cognitive processes," Trends in Cognitive Sciences, vol. 7, no. 12, pp. 553-559, Dec. 2003, doi: 10.1016/j.tics.2003.10.012.

[3] A. Oswal, P. Brown, and V. Litvak, "Synchronized neural oscillations and the pathophysiology of Parkinson's disease," Curr. Opin. Neurol., vol. 26, no. 6, pp. 662-670, Dec. 2013. doi: 10.1097/WCO.0000000000000034.

[4] Repp, Bruno H. 2005. "Sensorimotor Synchronization: A Review of the Tapping Literature." Psychonomic Bulletin and Review, vol. 12, no. 6, pp. 969-92, Dec. 2005, doi: 10.3758/BF03206433.

[5] Drake, C., Penel, A., and Bigand, E. (2000). "Tapping in time with mechanically and expressively performed music." Music Perception: An Interdisciplinary Journal, vol. 18, no. 1, pp. 1-23, Oct. 2000, doi: 10.2307/40285899.

[6] H.-H. Schulze, "The Error Correction Model for the Tracking of a Random Metronome: Statistical Properties and an Empirical Test," in Time, Action and Cognition: Towards Bridging the Gap, F. Macar, V. Pouthas, and W. J. Friedman, Eds. Dordrecht: Springer Netherlands, 1992, pp. 275-286.

[7] J. Mates, "A model of synchronization of motor acts to a stimulus sequence," Biol. Cybern., vol. 70, no. 5, pp. 463-473, Mar. 1994, doi: 10.1007/BF00203239.

[8] R. C. Schmidt and B. O'Brien, "Evaluating the Dynamics of Unintended Interpersonal Coordination," Ecological Psychology, vol. 9, no. 3, pp. 189-206, Sep. 1997, doi: 10.1207/s15326969eco0903_2.

[9] B. H. Repp and P. E. Keller, "Adaptation to tempo changes in sensorimotor synchronization: effects of intention, attention, and awareness.," The Quarterly journal 
of experimental psychology. A, Human experimental psychology, vol. 57, no. 3, pp. 499-521, 2004.

[10] E. W. Large and C. Palmer, "Perceiving temporal regularity in music," Cognitive Science, vol. 26, no. 1, pp. 1-37, 2002, doi: 10.1016/S0364-0213(01)00057-X.

[11] E. W. Large and J. F. Kolen, "Resonance and the perception of musical meter," Connection Science, vol. 6, no. 2-3, pp. 177-208, 1994, doi: 10.1080/09540099408915723.

[12] D. Temperley, "A Unified Probabilistic Model for Polyphonic Music Analysis," Journal of New Music Research, vol. 38, no. 1, pp. 3-18, Mar. 2009, doi: $10.1080 / 09298210902928495$.

[13] A. Bose, Á. Byrne, and J. Rinzel, "A neuromechanistic model for rhythmic beat generation," PLOS Computational Biology, vol. 15, no. 5, p. e1006450, May 2019, doi: 10.1371/journal.pcbi.1006450.

[14] J. D. Loehr, E. W. Large, and C. Palmer, "Temporal coordination and adaptation to rate change in music performance," Journal of Experimental Psychology: Human Perception and Performance, vol. 37, no. 4, pp. 1292-1309, 2011, doi: 10.1037/a0023102.

[15] J. D. Loehr and C. Palmer, "Temporal coordination between performing musicians," The Quarterly Journal of Experimental Psychology, vol. 64, no. 11, pp. 2153-2167, Nov. 2011, doi: 10.1080/17470218.2011.603427.

[16] J. D. Loehr, D. Kourtis, C. Vesper, N. Sebanz, and G. Knoblich, "Monitoring Individual and Joint Action Outcomes in Duet Music Performance," Journal of Cognitive Neuroscience, vol. 25, no. 7, pp. 1049-1061, Mar. 2013, doi: 10.1162/jocn_a_00388.

[17] Rasch, R. A. (1979). "Synchronization in performed ensemble music," Acustica, vol. 43, pp. 121-131.

[18] Shaffer, L.H. (1984). "Timing in solo and duet piano performances," Quarterly Journal of Experimentla Psychology, vol. 36, no. 4, pp. 577-595, Nov. 1984, doi: 10.1080/14640748408402180.

[19] L. Glass and M. C. Mackey, From Clocks to Chaos: The Rhythms of Life. Princeton University Press, 1988. 
[20] T. Saigusa, A. Tero, T. Nakagaki, and Y. Kuramoto, "Amoebae Anticipate Periodic Events," Phys. Rev. Lett., vol. 100, no. 1, p. 018101, Jan. 2008, doi: 10.1103/PhysRevLett.100.018101.

[21] M. Tachikawa, "A mathematical model for period-memorizing behavior in Physarum plasmodium," Journal of Theoretical Biology, vol. 263, no. 4, pp. 449-454, Apr. 2010, doi: 10.1016/j.jtbi.2010.01.005.

[22] N. Mosheiff et al., "Inheritance of Cell-Cycle Duration in the Presence of Periodic Forcing," Phys. Rev. X, vol. 8, no. 2, p. 021035, May 2018, doi: 10.1103/PhysRevX.8.021035.

[23] Hanson, F.E. (1982). "Pacemaker control of rhythmic flashing of fireflies," Carpenter, D. (ed.) Cellular Pacemaker, vol. 2, pp. 81-100. New York: John Wiely.

[24] Ermentrout, Bard. "Simulating, analyzing, and animating dynamical systems: a guide to XPPAUT for researchers and students." SIAM vol. 14, 2002.

[25] Edelstein-Keshet, Leah. "Mathematical Models in Biology". SIAM, 2005.

[26] J. C. Kim and E. W. Large, "Mode locking in periodically forced gradient frequency neural networks," Phys Rev E, vol. 99, no. 2-1, p. 022421, Feb. 2019, doi: 10.1103/PhysRevE.99.022421. 